\title{
The Fast and the Slow of Skilled Bimanual Rhythm Production: Parallel Versus Integrated Timing
}

\author{
Ralf T. Krampe, Reinhold Kliegl, \\ Ulrich Mayr, and Ralf Engbert \\ University of Potsdam
}

\author{
Dirk Vorberg \\ Technical University of Braunschweig
}

\begin{abstract}
Professional pianists performed 2 bimanual rhythms at a wide range of different tempos. The polyrhythmic task required the combination of 2 isochronous sequences ( 3 against 4 ) between the hands; in the syncopated rhythm task successive keystrokes formed intervals of identical (isochronous) durations. At slower tempos, pianists relied on integrated timing control merging successive intervals between the hands into a common reference frame. A timer-motor model is proposed based on the concepts of rate fluctuation and the distinction between target specification and timekeeper execution processes as a quantitative account of performance at slow tempos. At rapid rates expert pianists used hand-independent, parallel timing control. In alternative to a model based on a single central clock, findings support a model of flexible control structures with multiple timekeepers that can work in parallel to accommodate specific task constraints.
\end{abstract}

Polyrhythms have received considerable attention in the study of bimanual coordination because they require the simultaneous production of conflicting (non-harmonicallyrelated) motor sequences, like three beats in the left hand versus four beats in the right hand. The dominant finding emerging from a number of investigations is that temporal coupling prevents genuine hand independence, or the parallel timing control of the two sequences. Even trained individuals tend to rely on integrated timing control concatenating successive between-hands intervals to merge them into a common temporal reference frame (Klapp, 1979). Evidence for strong temporal coupling supports the notion of a central timer or clock (Wing \& Kristofferson, 1973b) that is called on to time responses for both hands. According to this framework, observed timing accuracy (variability) in bimanual rhythm tasks is mainly constrained by the temporal resolution of this central, unitary device.

In this study, we investigated professional pianists' timing accuracy in two tasks that either encouraged integrated or

Ralf T. Krampe, Reinhold Kliegl, and Ulrich Mayr, Department of Psychology, University of Potsdam, Potsdam, Germany; Ralf Engbert, Department of Physics, University of Potsdam; Dirk Vorberg, Institute for Psychology, Technical University of Braunschweig, Braunschweig, Germany.

This, research was initiated at the Max Planck Institute for Human Development and Education in Berlin, Germany and is now continued as part of the interdisciplinary project Formal Models of Cognitive Complexity at the Center for Cognitive Studies, University of Potsdam. The project is funded by Grant INK 12/A1 (Project B1) of the German Research Foundation. The friendly support of Yamaha Europe is acknowledged. We are indebted to Richard Jagacinski for helpful discussions during the initial stages of developing the models.

Correspondence concerning this article should be addressed to Ralf T. Krampe, Department of Psychology, University of Potsdam, P.O. Box 6015 53, D-14 415 Potsdam, Germany. Electronic mail may be sent to krampe@rz.uni-potsdam.de. parallel timing. We demonstrate that integrated timing is the predominant control mode at slower tempos and present a quantitative model that accounts for the observed performances across a wide tempo range. We then show that highly trained pianists can circumvent the constraints arising from temporal coupling and integrated timing through parallel, hand-independent timing control when performing at rapid tempos.

We first introduce integrated and parallel timing concepts and illustrate the tasks used in our investigation. We then describe the central clock model and the extension of the two-level timing framework toward bimanual performance. The focus of the following section is on the concept of timekeeper structures in timer-motor models of rhythmic performance. Finally, we review studies that used polyrhythmic tasks to directly address the issue of parallel versus integrated timing. We argue that earlier studies did not capture the full range of flexibility underlying expert timing capacities and detail our approach to the problem.

\section{Integrated and Parallel Timing}

In many studies of human movement timing, the participants' task is to repeatedly produce taps separated by identical (i.e., isochronous) intervals with a single finger or hand. In rhythmic (especially musical) performance this situation is the exception. At a minimum, successive keystrokes form groups and often intervals within these rhythmic groups differ in their target durations. Bimanual polyrhythms are special in that they require simultaneous production of conflicting (non-harmonically-related) isochronous motor sequences with the hands; they are a challenge even for trained musicians. Figure 1 illustrates the two tasks we used in our study for the example cycle duration of 1,200 $\mathrm{ms}$. The top panel depicts the three against four polyrhythm; the bottom panel shows our control task, which we call the "syncopated rhythm task." 


\section{Polyrhythm 3 Against 4}

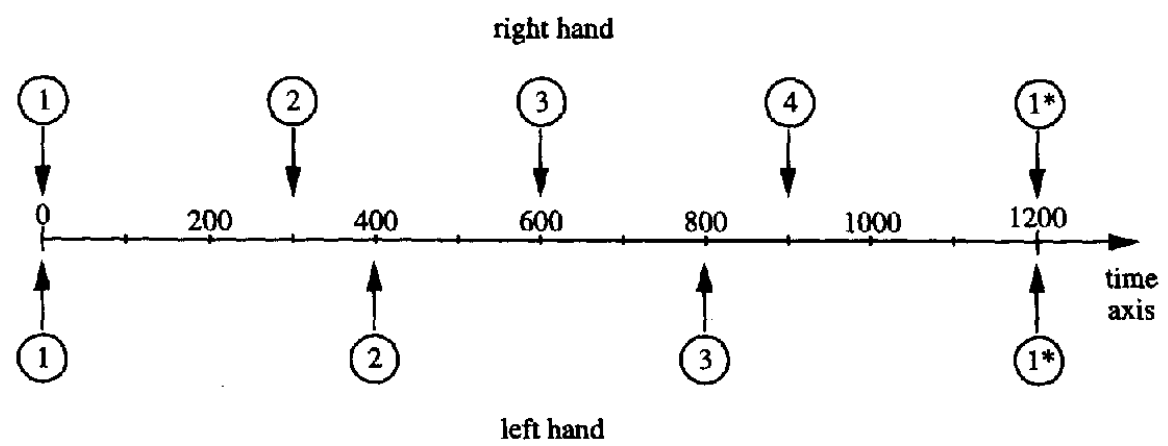

Syncopated Rhythm

right hand

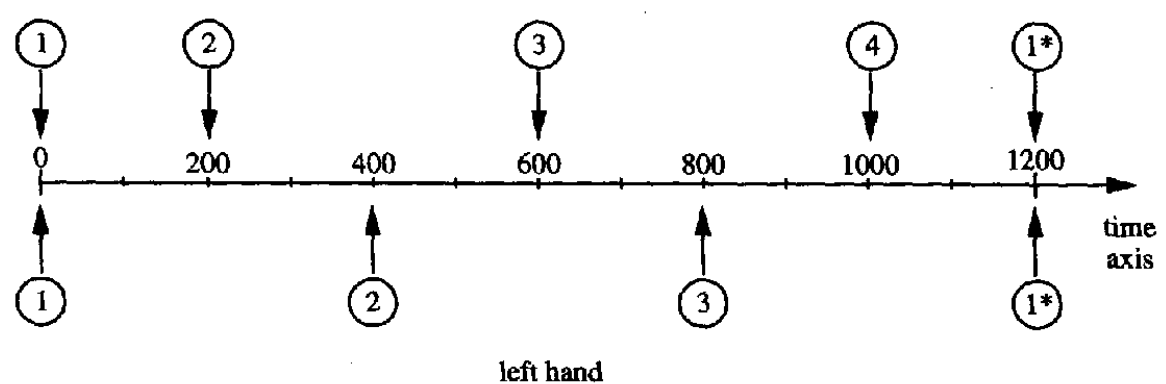

Figure 1. Schematic illustration of the optimal pattern of keystroke timing for one cycle of $1,200-\mathrm{ms}$ duration in the two tasks used in this study. The top panel illustrates the three (left-hand) against four (right-hand) polythythm. The bottom panel shows the syncopated thythm used as a control task. A rhythmic cycle in either task starts with simultaneous strokes; the sequence of strokes is identical for both tasks. Within-hands intervals are isochronous in the polyrhythm; all intervals including those between hands have identical (isochronous) target durations in the syncopated thythm task.

In bimanual rhythm production two problems arise: (a) to maintain the proper sequence of taps with the hands and (b) to produce intervals of the prescribed duration. In the two rhythms illustrated, the serial order of taps with the hands is identical: The first keystrokes with either hand occur simultaneously, and within cycles the hands alternate starting with the right hand. The difference between the thythms is their temporal structure. The polyrhythm requires the performance of three isochronous intervals in the left hand while simultaneously playing four isochronous intervals with the right hand; that is, the polyrhythm has an isochronous within-hands structure. In the syncopated rhythm all successive taps are separated by the same (isochronous) durations no matter which hand initiates or terminates a certain interval. In contrast, successive, nonoverlapping intervals in the polyrhythmic task form an anisochronous sequence of long (300 ms); short (100 ms); medium (200 ms), and medium, short, long intervals during a certain rhythmic cycle.

In its simplest form, integrated timing of these rhythms delineates a synchronous tap as well as six nonoverlapping intervals rather than four intervals for the right and three for the left hand. The characteristic feature of integrated timing is that the time point of a given tap in one hand (e.g., the second left-hand tap) mostly depends on its immediate predecessor (the second right-hand tap in our example) rather than on the preceding tap from the same hand. A more intuitive approach to integrated timing is to subdivide the cycle duration by the smallest interval to be timed and place successive taps from either hand on the appropriate beats. 
This is in fact a common exercise among musicians when they practice novel rhythmic patterns. For the syncopated rhythm, one can simply count from 1 (joint tap) up to 6 and place the proper tap on each counted beat. For the polyrhythm, this method requires counting 12 beats; if the simultaneous tap is at "one," successive right-hand taps must be placed on 4,7 , and 10. The two left-hand taps within the cycle are at 5 and 9.

Conscious counting, although, of course, only a method during initial practice at slower tempos, illustrates an important aspect: Timing a sequence of varying interval durations is more difficult than for an isochronous pattern and results in higher variability (Collier \& Wright, 1995; Povel, 1981; Vorberg \& Hambuch, 1984). From this perspective, the within-hands structure of the polyrhythm lends itself to timing out two isochronous sequences, one for each hand. This is exactly what parallel timing amounts to. Parallel control in the syncopated rhythm task is more difficult. The left within-hands intervals are identical in both rhythms; however, the right hand taken alone must perform an anisochronous sequence of short ( $200 \mathrm{~ms})$, long (400 ms), and long and short intervals in the syncopated rhythm task.

There is one further problem inherent to parallel timing: Even if individuals were able to maintain parallel control of two isochronous sequences, some form of temporal coupling of the hands is required to prevent the parallel sequences from drifting apart. Drifting apart eventually leads to errors in terms of violations of the serial order of taps from the hands. In integrated timing, the proper sequencing of the hands is guaranteed because fixed associations between the hands and time signals from a single, temporal reference frame can be established.

Thus far we have provided only a general outline of integrated and parallel timing. We now turn to a more detailed discussion of theoretical frameworks and the empirical studies motivated by them.

\section{The Central Clock Model and the Two-Level Timing Framework}

Wing and Kristofferson (1973a, 1973b) proposed a twolevel model of movement timing that distinguishes between a central clock or timer and a peripheral implementation level. The central timer triggers relevant effector systems at appropriate time points, which then execute responses with a certain motor delay. So conceived, the central timer is a multipurpose mechanism that is called on whenever the timing of behavior is critical with respect to external or internal constraints. Results of several studies suggest that the central clock mechanism constitutes a stable source of interindividual differences that are relevant to timing accuracy both in movement production tasks with different effectors and in perceptual judgment tasks (Ivry \& Hazeltine, 1995; Keele, Pokorny, Corcos, \& Ivry, 1985).

In their original approach to timing accuracy in repetitive tapping tasks, Wing and Kristofferson (1973a) conceptualized central timer (CT) and motor delays (MD) as uncorrelated, random variables across levels and within a series. The central timer generates successive intervals in an open-loop manner that is without reference to internal or external feedback. Given that successive observed intervals are separated by the identical motor event, the model accounts for the negative lag-1 autocorrelation frequently observed in repetitive movement tasks by random shifts in the motor delay component. The variance of the motor delays $(m)$ equals the absolute value of the lag-1 autocovariance calculated from a sequence of interkeystroke intervals:

$$
\operatorname{cov}\left(I_{n}, I_{n+1}\right)=-m .
$$

More generally, in the two-level model observed timing variability amounts to the sum of the variances independently contributed by central timer and motor delay components:

$$
\sigma^{2}(I)=\sigma^{2}(C T)+2 m .
$$

Under these assumptions, the observed variability can be decomposed into central clock variability and motor delay variance by simple subtraction (for a detailed description, see Vorberg \& Wing, 1996). If the model holds, lag-1 autocorrelations cannot be smaller (i.e., more negative) than -.5 and all higher order autocorrelations must be zero. These predictions were confirmed in Wing and Kristofferson's $(1973 a, 1973 b)$ original work and several studies thereafter. The basic task used in related studies involves the continuation paradigm, in which participants initially synchronize their repeated presses on Morse keys to a periodic auditory sound signal and subsequently continue to generate a series of unpaced responses. The critical analyses are usually performed on the responses generated during the continuation phase.

Strong support for the two-level timing model came from studies in which the target interval was varied across trials. The general finding was that the variance of the observed intervals increases as a function of target duration (Ivry \& Hazeltine, 1995; Wing, 1980; Wing \& Kristofferson, 1973b). Using the decomposition technique just described, Wing showed that this increase could be exclusively attributed to the central clock while the implementation component estimated from the variability of motor delays remained constant across tempos.

Several proposals have been made to extend the two-level timing framework toward bimanual performance (Vorberg \& Hambuch, 1978, 1984; Wing, 1982). The critical assumption is that the same central timing mechanism controls both hands and triggers appropriate responses at a lower level. We refer to this variant as the extended two-level timing framework. According to this framework, asynchronies between simultaneous keystrokes arise solely from differences between motor delays for the hands. Another intriguing consequence of two-level timing in the bimanual case relates to the alternation of the hands in a repetitive tapping task: Wing, Church, and Gentner (1989) found that the variability of within-hands intervals in the alternate tapping task was reduced compared with the unimanual case. The extended two-level timing framework accounts for this finding by assuming that intervals of only half the duration 
are timed at a central level in the alternate tapping condition as compared with the unimanual case.

\section{Timer-Motor Models}

The implicit assumption underlying most of the earlier work motivated from Wing and Kristofferson's (1973a, 1973b) model was that the central clock is a programmable device that can realize successive intervals by implementing appropriate target values. As a different approach, Vorberg and Hambuch $(1978,1984)$ introduced the idea of timekeeper structures as the basic concept underlying so-called "timer-motor models of rhythmic timing." Vorberg and Hambuch incorporated the two-level timing framework in their approach; timekeepers operate at a cognitive, presumably central, level and trigger responses after their specific target delay. The response is then implemented by the peripheral motor system with another delay. Different from a single, programmable clock, a timekeeper has one, and only one, specific target interval. So conceived, a timekeeper has a one-time purpose and resembles an egg clock rather than a programmable stopwatch. Timekeeper structures consist of multiple timekeepers that can differ in their target intervals and are specific to the rhythmic task under consideration.

The simplest form of timekeeper structure is a series of strictly concatenated timekeepers. For the rhythms depicted in Figure 1, this amounts to assuming a series of six timekeepers that have different target intervals in the polyrhythmic task. Each timekeeper performs its task and then starts its immediate successor in the structure. Strict concatenation is the strongest form of integrated timing in bimanual tasks because the hands are controlled with permanent reference to each other. Alternatively, we may assume two sets of timekeepers providing distinct control structures for the hands, thereby instantiating parallel timing. In hierarchical timekeeper models, superordinate timekeepers control timekeepers at lower levels, thereby inducing covariation among intervals controlled by these lower level timekeepers.

The first successful application of the timekeeper framework was an account of the phenomenon of rhythmic grouping. Vorberg and Hambuch (1978) instructed participants to group repetitive taps by placing each, every second, third, or fourth response on a pacing signal. They identified higher order positive covariances in the observed response series that coincided with the induced rhythmic grouping. A similar finding emerged in a later study by Vorberg and Hambuch (1984), in which participants generated isochronous and anisochronous rhythms. Isochronous performance corresponded to the assumptions of strict concatenation, and the variability of intervals in these tasks was smaller than for the anisochronous rhythms. As an alternative to the serial autocovariance function applied in the original Wing and Kristofferson (1973a) work, Vorberg and Hambuch developed an approach in which covariances among intervals are calculated across a series of rhythmic cycles. The authors showed that the positive higher order covariances in the anisochronous sequences did not support any of the hierarchical timing models considered but were the result of systematic differences in mean realized intervals that participants introduced in their rhythmic grouping; strictly sequentially organized timekeepers (i.e., concatenation) could not be rejected on the basis of these findings. As a consequence, Vorberg and Hambuch (1984) suggested that the temporal properties of observed behavior reflect the joint operation of separate systems for serial order control and the actual timing control during execution.

More recently, Vorberg and Wing (1996) elaborated this approach into a model they called the "rhythm program hypothesis." The rhythm program hypothesis provides a general framework for tasks with an explicit rhythmic component and is oriented toward musical (i.e., expressive) performance. At the core of the model is the distinction between parameter specification of target intervals for timekeepers and their execution. The term parameter specification refers to the programming of the target intervals for different timekeepers as specified in the underlying rhythm program. Similar to the concept of motor programs, a rhythm program is an abstract representation of the serial order of events in an action sequence that translates into a hierarchy of adjustable timekeepers when implemented at the time of performance. At the top-most level, information about the current rate (overall performance tempo) enters into the parameter specification process. Rate is subject to fluctuation, which can be regarded as the deviation of the momentary tempo from the intended mean. Subsequently, timekeepers at different hierarchy levels determine reference durations, which are then propagated as target values for timekeepers in the next lower level of the hierarchy. Naturally, rate fluctuation will have an effect on all timekeepers directly relying on the rate for their target specification. The effect is to induce positive covariation among the actual target values for the respective intervals. Eventually, the serial order control system translates a motor program into target intervals for a set of concatenated timekeepers that can then be executed in a strictly sequential fashion. These timekeepers realize the computed target intervals with a certain variability, a process that in turn adds to the variance already caused at the target specification (i.e., programming) stage. In their theoretical analyses, Vorberg and Wing showed that rhythm program models can, in principle, account for several empirical findings that were troublesome for earlier models like those developed in the context of the invariant-relative timing notion originally proposed in the context of general motor programming (for discussion, see Gentner, 1987; Heuer, 1988, Heuer, 1991).

In summary, the assumption of a single central clock that is reprogrammed if intervals differ in their durations cannot account for the positive covariance patterns obtained from rhythmic performance. Timer-motor models adopt the extended two-level timing framework, but they do not commit to the existence of one single clock as an exclusive timing device. The issue of hand-independent timing control can be recast in the timer-motor context: The critical question is whether integrated or parallel timekeeper structures underlie performance. Before we detail our approach, we turn to studies that directly addressed the issue of hand independence in the investigation of polyrhythmic performance. 


\section{Timing in Polyrhythmic Performance}

The results from a number of studies suggest that even trained individuals integrate different temporal trajectories into a common time frame for the hands (Deutsch, 1983; Jagacinski, Marshburn, Klapp, \& Jones, 1988; Klapp, 1979; Klapp et al., 1985; Summers, Rosenbaum, Burns, \& Ford, 1993). Neither extensive practice of more than 4,000 cycles in a polyrhythmic task (Summers, Ford, \& Todd, 1993) nor training the hands separately before simultaneous performance (Klapp et al., 1985; Klapp, Nelson, \& Jagacinski, 1998; Summers \& Kennedy, 1992) induced temporal decoupling. The only exception from a long list of findings suggesting integrated timing of some sort is a pioneering study by Shaffer (1981). In analyzing one top-level pianists' repeated performances of a musical piece with polyrhythmic structure, Shaffer observed that pianists systematically varied which of the hands was leading in relation to the other. Shaffer's analyses of covariance provided indications for independent timing variation of the hands.

The earliest attempt we know of to systematically compare the variance-covariance structures derived from alternative control models for polyrhythmic performance was described by Jagacinski et al. (1988). They used a synchronization paradigm to investigate musically trained (pianists) participants' timing of a 2 against 3 polyrhythm at a cycle duration of $1,300 \mathrm{~ms}$ (i.e., 433-ms intervals for the faster hand). The analyses of covariance structures supported an integrated timing model. In addition, Jagacinski et al. observed positive covariances that they took as evidence for a hierarchical multiplicative control structure.

The model proposed by Summers, Rosenbaum, et al. (1993) is a special variant of integrated timing, namely a two-tiered model in which the slower hand (i.e., the hand producing 3 beats in a 3:4 rhythm) is subordinate to the faster hand. According to the model, a single counter mechanism (similar to the central clock) delineates intervals within a cycle and triggers responses at the proper occasions. Summers et al. argued that individuals focus their attention on the faster hand to produce a steady beat as a time base, whereas they insert the slow hand responses between two fast-hand beats with less temporal precision. One implication of this model is that taps from the slow hand tend to be placed in the middle between two fast-hand taps rather than at their appropriate positions. Variability of slower hand intervals was indeed higher than with the faster hand in this study, and participants showed the predicted shifts in tap placements. However, these effects were typical of nonmusicians' performance and did not show systematically in the musically trained participants.

The most thorough modeling approach to polyrhythmic performance was provided by Pressing, Summers, and Magill (1996). In their study three highly trained percussionists produced 3:4 and 4:3 polyrhythms on Morse keys at a cycle duration of $1,800 \mathrm{~ms}$ while receiving a synchronization signal over headphones. They instructed their participants to selectively apply different production models corresponding to a figure-ground distinction for the 3 and the 4 beat, respectively. According to Pressing et al., a central clock process underlies the production of the ground beat. The figure emerges from timekeepers delineating intervals that have their starting points at the previous ground beat. Pressing et al. made several assumptions about covariation between higher and lower level timekeepers and differences between figure and ground motor delays. Under these assumptions, their general hierarchical model explained critical aspects of their data. The figure-ground distinction corresponds to musicians' description of their own polyrhythmic performance. In the model proposed by Summers, Rosenbaum, et al. (1993) subordination of the slow under the fast hand is attributed to intrinsic difficulties and thus a necessary consequence of integrating the hands. Different from this approach, Pressing et al. demonstrated that experts can apply different mental models even under varying hand assignments.

In summary, the prevailing conclusion from several studies is that even musically trained individuals rely on integrated timing of the hands when performing polyrhythmic tasks. Although polyrhythms have enjoyed considerable attention as an object of empirical investigation, relatively few researchers have attempted to explicitly model the variance-covariance structure implied by related theoretical models. Those researchers reported evidence violating the original Wing-Kristofferson model. Consequently, some variant of an integrated timer-motor model was used along with specific assumptions to account for the findings. Although these studies identified shortcomings of the WingKristofferson model, they did not lead to a coherent alternative. We trace the interpretative ambiguities to two sources: First, with the notable exception of the studies by Pressing et al. (1996) and Shaffer (1981), the expertise level of participants ranged from little to "normal" musical skills. Second, past research focused on polyrhythmic performance at a single tempo or few tempos. These restrictions are a problem if the goal is to capture the full range of human timing capacities.

Our approach was to investigate performance characteristics in highly accomplished experts under task conditions that maximally challenged their timing capacities. A central notion in theories of expertise is that highly skilled individuals can circumvent the processing limitations constraining normal performance by domain-specific mechanisms (Chase \& Ericsson, 1982; Ericsson \& Charness, 1994; Salthouse, 1991). Previous research has demonstrated that the amount of training necessary for an individual to acquire related mechanisms is huge; it surpasses by orders of magnitude the practice usually administered under laboratory conditions or the amount of training typical for amateurs in a domain (Ericsson, Krampe, \& Tesch-Römer, 1993; Krampe \& Ericsson, 1996). From the expertise perspective, hand interdependence and the increase of variability with target duration constitute performance constraints. The relevance of these constraints depends on tempo and the rhythmic complexity imposed by a certain task. Our basic tenet is that highly skilled individuals have developed timing capacities that comprise both integrated and parallel timing and that they can fine-tune these mechanisms in adaptation to performance constraints. 


\section{Overview of the Experiment}

Our rationale was to use tasks (see Figure 1) that either encouraged integrated or parallel timing, respectively. We argued before that the polyrhythmic pattern provides the more promising performance context for eliciting hand independence or parallel timing control because of its isochrony of within-hands intervals. ${ }^{1}$ The second rationale behind the design of our study relates to another performance constraint, tempo, and we now consider the implications for our predictions in more detail.

Although parallel control supports isochronous withinhands timing (at least in the polyrhythmic task), it does not automatically guarantee the proper sequencing of keystrokes coming from different hands. To this end, a coordination (coupling) of the parallel timekeepers is required. Whatever the exact nature of this coupling or correction process is, its functionality is severely constrained by a well-documented phenomenon that we discussed earlier, the dependence of variability on target duration. For example, at slower tempos the time window for coupling timekeepers working in parallel becomes increasingly larger and more difficult for the cognitive system to predict. The obvious consequence is that the hands will drift apart if the variabilities for within-hands intervals grow progressively. From this perspective, it seems plausible that the human motor system controls long within-hands intervals by concatenating timekeeper signals for shorter between-hands intervals to constrain the increase of variability. Therefore, integrated control is also the more likely candidate for polyrhythmic timing at slower tempos in the present study. Parallel timing, if at all possible, would be restricted to fast performance rates.

We now consider the potential benefits of parallel timing at rapid tempos with respect to the differences between our two tasks. For typing skills, Gentner (1988) proposed that experts have optimized the efficiency of their cognitive processes to a degree that their performance is almost exclusively constrained by biomechanical limitations. The strongest biomechanical constraint in our tasks arises from repeated taps with the same (right) finger. Alternate tapping is much faster than single-hand tapping, and this advantage is even more pronounced in expert pianists than in amateurs (Krampe \& Ericsson, 1996). Figure 1 reveals that the repeated taps with the same (right) finger (1-2 and 4-1*) involve longer intervals in the polyrhythmic $(300 \mathrm{~ms}$ in our example) than the syncopation control task $(200 \mathrm{~ms})$. If expert musicians were to adapt to rapid performance constraints by applying parallel timing control, the benefits would be greatest in the polyrhythmic task. Ultimately, this implies that the rhythmically much simpler task becomes more difficult than the polyrhythmic task at rapid rates.

In summary, we expected some form of integrated timing in both tasks at slower tempos. Our goal was to identify the specific version of integrated model that captures performance in tasks of diverse rhythmic complexity for a large range of different tempos. Our hypothesis for rapid performance was that parallel timing can emerge in highly trained individuals if dictated by specific task constraints, most notably in polyrhythmic performance.

\section{Timekeeper Organizations in Integrated and Parallel Models}

Both models we propose are instances of the simplest form of timekeeper organization: strict concatenation. $\mathrm{Nu}$ merous variants of parallel and integrated models for the two rhythm tasks are conceivable once we include hierarchical timekeeper structures; Jagacinski et al. (1988) identified 30 variants alone for the simpler 3 against 2 polyrhythm. Hierarchical timekeeper structures can be identified in terms of systematic deviations in the covariance matrices from the simple models we propose. In that respect, our proposal remains subject to evaluation against the data. Throughout the rest of this article, we refer to the strictly concatenated versions in our usage of the terms integrated and parallel models, respectively.

Figure 2 shows the integrated (top panel) and the parallel timing control models (middle panel) for the 3 against 4 polyrhythm used in our study. Note that the modeling approach described in the following is perfectly analogous for both tasks. We have omitted the motor delays in the illustrations for convenience. The bottom panel illustrates the observed intervals and their correspondence to the hypothetical timekeepers in the depicted timing control models. In Figure 2 we use small letters to denote occurrences of keystrokes and capital letters for intervals. We refer to the successive keystrokes within the right hand as $r_{1}$, $r_{2}, r_{3}$, and $r_{4}$ and those within the left hand as $l_{1}, l_{2}$, and $l_{3}$.

A simplifying strategy in earlier approaches (Jagacinski et al., 1988) was to average the time points of the joint keystrokes at the beginning of each cycle to obtain a common reference point for the first and last intervals within the cycle. We followed the alternative suggestions in Pressing et al. (1996) and considered all seven intervals in each rhythmic cycle that can be directly observed, including the asynchrony between the first keystroke for both hands. We define the asynchrony (denoted by $I_{0}$ in Figure 2 ) as the difference $l_{1}-r_{1}$ between the onsets of the first keystrokes with the left and the right hand, such that the value becomes negative if the left onset precedes the right one. The remaining between-hands intervals within each rhythmic cycle $\left(I_{1}-I_{6}\right)$ are defined accordingly; for example, $I_{1}=$ $r_{2}-l_{1}, I_{2}=l_{2}-r_{2}$, and so on. Note that by these definitions $I_{1}$ is always between hands, even if $I_{0}$ is negative. The right and left within-hands intervals are defined analogously; for example, $R_{1}=r_{2}-r_{1}$, and $\mathrm{L}_{2}=l_{3}-l_{2}$.

The integrated model consists of a series of concatenated timekeepers (denoted A, B, C, D, E, and F) that control six nonoverlapping intervals between successive keystrokes.

\footnotetext{
1 We restrict the meaning of "hand independence" to variation at the level of timekeepers (i.e., above the motor delay level) independent of the other hand. In principle, hand independence could also occur at the level of force control, which may actually be more critical in musical phrasing of polyphonic music, or at the level of movement trajectories. Other authors, such as Pressing et al. (1996), argued that subordinate timing relations (i.e., if the control of one hand is done relative to the other) constitutes a case of hand independence.
} 


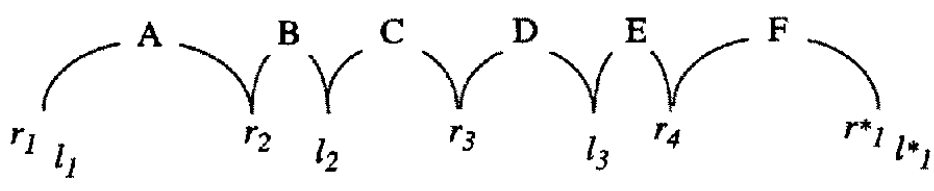

Parallel Timing Control
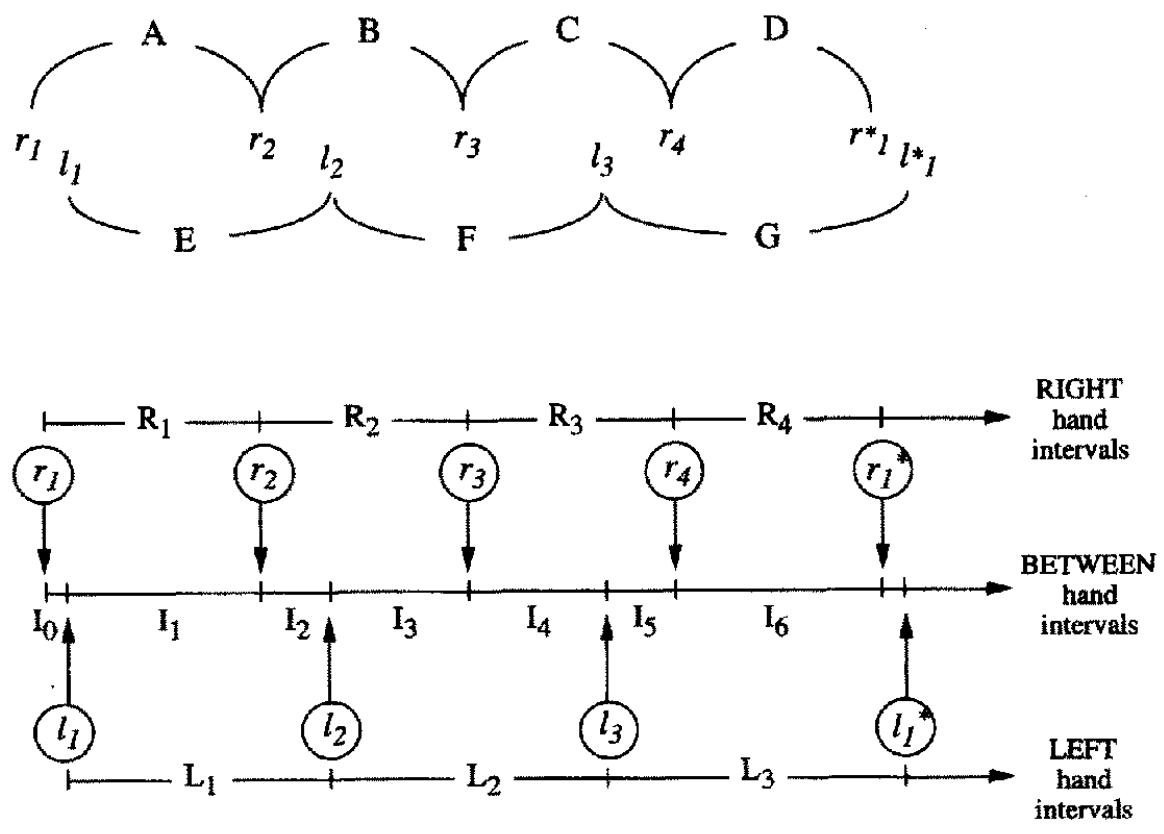

Time Axis and Intervals

Figure 2. Integrated and parallel timing control structure and intervals considered in the modeling of covariance structures. Small letters in the two upper panels denote keystrokes with the right hand $\left(r_{1}\right.$ to $\left.r_{4}\right)$ and the left hand $\left(l_{1}\right.$ to $\left.l_{3}\right)$, respectively. The bottom panel illustrates the observed intervals. $\mathrm{I}_{0}$ is the asynchrony of the joint strokes of both hands at the beginning of each cycle. The integrated model (top panel) presumes a common temporal reference frame for the hands. A set of serialized timekeepers (A-F) control single intervals (denoted $I_{1}$ to $I_{6}$ in the bottom panel) regardless of starting and terminating hand. The parallel model (middle panel) instantiates hand independence: Timekeepers $A-D$ control right-hand intervals (denoted $R_{1}$ to $R_{4}$ in the bottom panel), and timekeepers $E-G$ control left-hand intervals (denoted $\mathrm{L}_{1}$ to $\mathrm{L}_{3}$ in the bottom panel).

The critical feature of integrated timing is that timekeepers perform their task irrespective of whether related intervals are delimited by right- or the left-hand keypresses or by simultaneous keypresses as it is the case for the termination of $\mathbf{I}_{6}$. Concatenation implies that a given timekeeper performs its function after which it triggers the next one and so on. Timekeepers $B$ to $F$ determine intervals $I_{2}$ to $I_{6}$; timekeeper A controls the interval $I_{1}$.
The parallel timing model consists of two sets of timekeepers, one for each hand. Timekeepers A, B, C, and D, delineate right-hand intervals $R_{1}$ to $R_{4}$, whereas $E, F$, and $G$ time out left-hand intervals $L_{1}$ to $L_{3}$. Within each set, timekeepers are again concatenated. Note that timekeepers denoted by the same capital letters do not have identical target values in different models or tasks.

The bottom panel in Figure 2 shows that each task 
comprises seven $\left(\mathrm{I}_{0}\right.$ to $\mathrm{I}_{6}$ ) observable, nonoverlapping intervals. The triangular variance-covariance matrix thus has 28 cells. We next develop the covariance predictions from the integrated and the parallel models in two separate sections, starting with the integrated model. We organize each section into (a) covariances including the asynchrony; (b) covariances between intervals $I_{1}$ to $I_{6}$; and (c) variances of intervals $I_{1}$ to $I_{6}$. Both models can be framed into the context of the rhythm program hypothesis, and we also rely on the techniques for deriving the equations described in Vorberg and Wing's (1996) original work, where interested readers can find further details. The Appendix includes examples for the derivations. We provide a summary and contrast of model predictions at the end of this section.

\section{Covariance Structure Predicted by the Integrated Model}

Asynchrony and covariances with $I_{1}$ to $I_{6}$. Given that timekeeper $F$ triggers both hands simultaneously, the asynchrony $I_{0}$ is just the difference between the motor delays for the right $\left(M D_{R}\right)$ and left $\left(M D_{L}\right)$ hands. Under these assumptions, we can directly derive the prediction for the variance of $\mathrm{I}_{0}$, denoted by $\sigma_{0}^{2}$ in the following, using the formula for the variance of differences (see the Appendix, Equation A3):

$$
\sigma_{0}^{2}=m_{R}+m_{L}-2 \operatorname{cov}\left(M D_{R}, M D_{L}\right),
$$

where $m_{R}$ and $m_{L}$ denote motor delay variances for the hands. Because of the assumption of uncorrelated motor delays the final (covariance) term of Equation 3 reduces to zero that is, $\sigma_{0}^{2}$ is just the sum of motor delay variances for the hands. Following the extended two-level framework, we expect invariance of motor delays with tempo and this prediction should likewise apply to $\sigma_{0}^{2}$. Covariances between the asynchrony and all nonadjacent intervals should be zero. According to the integrated model, the covariances with intervals adjacent to $I_{0}$ must be negative and also provide direct measures of motor delay variances (Equation 1). The bottom panel in Figure 2 shows that the covariance between $I_{0}$ and $\mathrm{I}_{1}$ must reflect left-hand motor delay variances. Likewise, the covariance between the last interval in the cycle $\left(\mathrm{I}_{6}\right)$ and the following asynchrony $\left(\mathrm{I}_{0}\right.$ at cycle-lag 1$)$ is a direct reflection of the motor delay variance for the right hand.
Covariances between $I_{I}$ to $I_{6}$. We assume that the target intervals for timekeepers $A$ to $F$ are specified (i.e., programmed) with reference to a rate parameter $\boldsymbol{X}$, which assumes a constant value $x$ for a given rhythmic cycle. We express the expected rate $E(X)$ as the mean realized cycle duration or cycle period. Furthermore, we assume that $X$ fluctuates across cycles with variance $\sigma_{X}^{2}$. If we assume that the timekeepers properly realize their mean target intervals on average (i.e., the mean observed intervals correspond to the mean target intervals), we can scale the target intervals in proportions of the cycle duration $X$, such as timekeeper $B$ has a mean target interval of $b E(X)$ with $b=E\left(I_{2}\right) / E(X)$. For a constant proportion $b$, the effect of rate fluctuation $\sigma_{X}^{2}$ on a certain interval is proportional to its target duration, that is, the standard deviation of the target interval for timekeeper $B$ equals $b \sigma_{X}$ (see Equation A1).

The assumption of an underlying rate in the integrated model results in the prediction of positive covariances between intervals controlled by timekeepers that use the same rate parameter as input for their target specification. For two timekeepers $A$ and $B$ realizing mean target durations of $a E(X)$ and $b E(X)$, respectively, the covariance of their target intervals $A$ and $B$ for constant proportions $a$ and $b$ equals

$$
\operatorname{cov}(A, B)=a b \sigma^{2}(X)
$$

Note that the prediction of positive covariation attributable to rate fluctuation equally applies to adjacent and nonadjacent interval pairs. For adjacent intervals the effects of shared motor delays must be taken into account. In their development of the thythm programming framework, Vorberg and Wing (1996) took a more general approach by allowing both error propagation and rate fluctuation. This means that the proportions specified for the target intervals can vary within trials for a specific interval. Our model is more restricted in that we assume that the target intervals are constant proportions of cycle durations within each trial. Note that this does not imply that target intervals are constants because they still vary as a function of rate fluctuation. The covariance matrix resulting from the assumption of rate fluctuation in the integrated model is shown in Table 1.

The predicted effects of rate fluctuation are proportional

Table 1

Covariances Between Intervals Predicted by the Integrated Model

\begin{tabular}{lcccccc}
\hline $\begin{array}{l}\text { Interval } \\
(\mathrm{I})\end{array}$ & $\mathrm{I}_{1}$ & $\mathrm{I}_{2}$ & \multicolumn{1}{c}{$\mathrm{I}_{3}$} & $\mathrm{I}_{4}$ & $\mathrm{I}_{5}$ & $\mathrm{I}_{6}$ \\
\hline $\mathrm{I}_{0}$ & $-m_{L}$ & 0 & 0 & 0 & 0 & 0 \\
$\mathrm{I}_{1}$ & & $a b \sigma_{X}^{2}-m_{R}$ & $a c \sigma_{X}^{2}$ & $a d \sigma_{X}^{2}$ & $a e \sigma_{X}^{2}$ & $a f \sigma_{X}^{2}$ \\
$\mathrm{I}_{2}$ & & & $b c \sigma_{X}^{2}-m_{L}$ & $b d \sigma_{X}^{2}$ & $b e \sigma_{X}^{2}$ & $b f \sigma_{X}^{2}$ \\
$\mathrm{I}_{3}$ & & & $c d \sigma_{X}^{2}-m_{R}$ & $c e \sigma_{X}^{2}$ & $c f \sigma_{X}^{2}$ \\
$\mathrm{I}_{4}$ & & & & $d e \sigma_{X}^{2}-m_{L}$ & $d f \sigma_{X}^{2}$ \\
$\mathrm{I}_{5}$ & & & & & $e f \sigma_{X}^{2}-m_{R}$ \\
\hline
\end{tabular}

Note, $\quad \sigma_{X}^{2}=$ Rate fluctuation (variance of $X$ ); $\sigma_{X}^{2}=q_{0}+q_{1} E(X)+q_{2} E^{2}(X) ; a, b, c, d, e$, and $f=$ expected proportions of cycle duration $X$ realized by six concatenated timekeepers $A, B, C, D, E$, and $F ; m_{R}$ and $m_{L}=$ variance of motor delays for right and left hands, respectively. 
to the mean interval durations, that is, in the polyrhythmic task, they should be largest for the two long intervals $I_{1}$ and $I_{6}$, and the smallest for $I_{2}$ and $I_{5}$, the two shortest intervals. We assume that rate fluctuation by itself depends on the overall performance tempo. To model the effects of rate fluctuation across tempos, we must add assumptions about the relation between $\sigma_{X}^{2}$ and $X$. To keep the model general, we use a second-order polynomial to describe this relation. This includes both Poisson and Weber-type dependencies as potential cases:

$$
\sigma_{X}^{2}=q_{0}+q_{1} E(X)+q_{2} E^{2}(X) .
$$

Variances of $I_{I}$ to $I_{\sigma}$. According to the rhythm program hypothesis, the observed variability of a certain interval reflects the programming process plus the variability introduced when timekeepers actually execute their target intervals. We use a second-order polynomial as well to describe the relation between execution variance and interval duration. As an example, consider interval $I_{2}$ controlled by timekeeper $B$ in the integrated model. Again, we express the mean target duration of $B$ in proportions of $X$, the cycle duration. Given that $X$ takes on a value of $x$, the target interval of $B$ is $b x$; we assume the variance of $I_{2}$ to be

$$
\sigma_{X}^{2}\left(I_{2} \mid X=x\right)=p_{0}+p_{1} b x+p_{2}(b x)^{2} .
$$

Given that the rate is not a constant (i.e., the target intervals vary as described earlier), the variance caused during timekeeper execution must be conditionally dependent on $\sigma_{x}^{2}$. The equation can be derived on the basis of conditional expectation (Vorberg \& Wing, 1996):

$$
\sigma^{2}\left(I_{2}\right)=\left(1+p_{2}\right) b^{2} \sigma_{X}^{2}+p_{0}+p_{1} b E(X)+p_{2} b^{2} E^{2}(X) .
$$

As before, the variances contributed by motor delays must be added. Table 2 shows the variance predictions for the intervals $I_{0}$ to $I_{6}$ in the integrated model. The variability caused by the target specification process is enclosed in brackets. The $p$ parameters describe the execution variance as a function of mean cycle duration. The asynchrony $I_{0}$ is not controlled by a timekeeper; it depends only on motor delay variances.

Because the timekeeper intervals are independent random variables, it follows that inaccuracies during the execution of target intervals do not change the covariances between different intervals (see Table 1). The positive covariation among observed intervals is a result of the rate fluctuation affecting only target interval specification.

\section{Covariance Structure Predicted by the Parallel Model}

Asynchrony and covariances with $I_{1}$ to $I_{6}$. The timekeeper organization depicted in the middle panel of Figure 2 resembles two integrated (strictly concatenated) models working in parallel. We assume that the hands "run freely" within a rhythmic group that they are basically timed in a within-hands concatenated fashion. We argued before that parallel timing requires some coupling or phase correction at the beginning of the cycle or else the hands will drift apart leading to a violation of the serial order of keystrokes. So conceived, the coupling process is basically an error correction process tuned to the goal of keeping the asynchrony small. No matter how such a process is conceived of in detail, the variance of the asynchrony must take on values over and above the sum of the two motor delay variances that contribute to $\sigma_{0}^{2}$ in the first place. Naturally, the variance introduced by error correction cannot be independent of motor delays and timekeeper variances for each hand under these assumptions. At a more general level, the magnitude of $\sigma_{0}^{2}$ must reflect the difficulty of coupling.

The pattern of covariances between $I_{0}$ and intervals $I_{1}$ to $I_{6}$ can be illustrated by a mental simulation using Figure 2 . Assume a lengthening of $\mathrm{I}_{0}$ : Everything else being equal, under the assumption of strict concatenation for withinhands intervals, this has the effect of shifting the left-hand series in time relative to the right-hand series. Betweenhands intervals terminated by the left hand (i.e., $I_{2}$ and $I_{4}$ ) become larger (i.e., positive covariation with $\mathrm{I}_{0}$ ), whereas between-hands intervals terminated by the right hand (i.e.,

\begin{tabular}{|c|c|c|c|}
\hline \multirow{2}{*}{$\begin{array}{l}\text { Observed } \\
\text { interval (I) }\end{array}$} & \multicolumn{2}{|l|}{ Predicted variance } & \multirow[b]{2}{*}{ Timekeeper } \\
\hline & Timekeeper execution + target specification & Motor delays & \\
\hline $\begin{array}{l}I_{0} \\
I_{1} \\
I_{2} \\
I_{3} \\
I_{4} \\
I_{5} \\
I_{6}\end{array}$ & $\begin{array}{l}\left(1+p_{2}\right)\left[a^{2} \sigma^{2}(X)\right]+p_{0}+p_{1} a E(X)+p_{2} a^{2} E^{2}(X)+ \\
\left(1+p_{2}\right)\left[b^{2} \sigma^{2}(X)\right]+p_{0}+p_{1} b E(X)+p_{2} b^{2} E^{2}(X)+ \\
\left(1+p_{2}\right)\left[c^{2} \sigma^{2}(X)\right]+p_{0}+p_{1} c E(X)+p_{2} c^{2} E^{2}(X)+ \\
\left(1+p_{2}\right)\left[d^{2} \sigma^{2}(X)\right]+p_{0}+p_{1} d E(X)+p_{2} d^{2} E^{2}(X)+ \\
\left(1+p_{2}\right)\left[e^{2} \sigma^{2}(X)\right]+p_{0}+p_{1} e E(X)+p_{2} e^{2} E^{2}(X)+ \\
\left(1+p_{2}\right)\left[f^{2} \sigma^{2}(X)\right]+p_{0}+p_{1} f E(X)+p_{2} f^{2} E^{2}(X)+\end{array}$ & $\begin{array}{c}m_{R}+m_{L} \\
m_{R}+m_{L} \\
m_{R}+m_{L} \\
m_{R}+m_{L} \\
m_{R}+m_{L} \\
m_{R}+m_{L} \\
2 m_{R}\end{array}$ & $\begin{array}{l}\text { A } \\
\text { B } \\
\text { C } \\
\text { D } \\
\text { E } \\
\text { F }\end{array}$ \\
\hline
\end{tabular}

Table 2

Variances of Observed Nonoverlapping Intervals Predicted by the Integrated Model

Note. $E(X)=$ Expected rate expressed as cycle duration (sum $\mathrm{I}_{0}-\mathrm{I}_{6}$ ); $\sigma_{X}^{2}=$ rate fluctuation (variance of $X$ ); $\sigma_{X}^{2}=q_{0}+q_{1} E(X)+q_{2} E^{2}(X) ; a, b, c, d, e$, and $f=$ expected proportions of cycle duration $X$ controlled by six concatenated timekeepers A, B, C, D, E, and F and realized in between-hands intervals; $p_{0}, p_{1}$, and $p_{2}=$ parameters specifying the relation between variability during timekeeper execution and tempo; $m_{R}$ and $m_{L}=$ variance of motor delays for right and left hand, respectively. 
$I_{1}, I_{3}$, and $I_{5}$ ) are shortened (negative covariation with $I_{0}$ ). The last interval $\mathrm{I}_{6}$ remains unaffected because it is the only genuine within-hands interval among $\mathrm{I}_{0}$ to $\mathrm{I}_{6}$.

To formally derive the covariance predictions for the parallel model, it is important that intervals $I_{1}$ to $I_{5}$ are no longer directly controlled by individual timekeepers but emerge as differences between timekeepers controlling within-hands intervals (see Figure 2). Table 3 illustrates these relations.

Note that $I_{0}$ appears in the equations for intervals $I_{1}$ to $I_{5}$. As a first approximation, we assume zero correlations between the asynchrony and the rate parameter $X$. Under this assumption, the covariances between the asynchrony and sum terms for intervals $I_{1}$ to $I_{5}$ shown in the right-most column of Table 3 reduce to the covariance of the asynchrony with itself, which is just $\sigma_{0}^{2}$. However, the sign of $\sigma_{0}^{2}$ in the covariance predictions alternates depending on the interval considered: The parallel model predicts negative covariances between $I_{0}$ with $I_{1}, I_{3}$, and $I_{5}$; covariances with $I_{2}$ and $\mathrm{I}_{4}$ are predicted to be positive. In addition, we must take the effect of shared motor delays between $I_{0}$ and $I_{2}$ into account. Finally, the parallel model predicts zero covariances between the asynchrony and $\mathrm{I}_{6}$, the only observable interval that is directly controlled by a timekeeper according to the model and hence does not have the asynchrony in its predictive equation.

Covariances between $I_{1}$ to $I_{6}$. Table 4 shows the covariance matrix for the polyrhythmic task predicted by the parallel model. Each prediction comprises three terms: a covariation with $\sigma_{0}^{2}$, the effect of rate fluctuation, and, for adjacent intervals, the effect of motor delays. We provide a full-size example for the covariances between $\mathrm{I}_{2}$ and its successors in the Appendix.

Table 4 shows that in the parallel model, the asynchrony affects all covariances among intervals $I_{1}$ to $I_{5}$. Although rate fluctuation causes positive covariance, negative and positive covariation is introduced by $\sigma_{0}^{2}$ and this is also true for nonadjacent interval pairs. The sign of the covariance introduced by $\sigma_{0}^{2}$ must alternate when the covariances of a given interval with its successors are considered. For the

Table 3

Relation Between Observed (Nonoverlapping) Intervals and Overlapping Target Intervals for Within-Hands Timekeepers in the Parallel Model

\begin{tabular}{cl}
\hline $\begin{array}{c}\text { Observed between- } \\
\text { hands interval (I) }\end{array}$ & $\begin{array}{c}\text { Expected durations for } \\
\text { between-hands intervals }\end{array}$ \\
\hline $\mathrm{I}_{0}$ & 0 \\
$\mathrm{I}_{1}$ & $A-\mathrm{I}_{0}$ \\
$\mathrm{I}_{2}$ & $E-A+\mathrm{I}_{0}$ \\
$\mathrm{I}_{3}$ & $A+B-E-\mathrm{I}_{0}$ \\
$\mathrm{I}_{4}$ & $E+F-A-B+\mathrm{I}_{0}$ \\
$\mathrm{I}_{5}$ & $A+B+C-E-F-\mathrm{I}_{0}$ \\
$\mathrm{I}_{6}$ & $D$ \\
\hline
\end{tabular}

Note. $A, B, C$, and $D=$ concatenated timekeepers A, B, C, and D for four successive right-hand intervals. Target durations are $A=$ $a E(X), B=b E(X)$, and so on. $E, F$, and $G$ control left-hand intervals.

aDurations expressed as sums of target durations for within-hands intervals. complete matrix, the covariances between different pairs of intervals $\mathrm{I}_{0}$ to $\mathrm{I}_{5}$ form a checkerboard pattern of positive and negative effects of $\sigma_{0}^{2}$.

The notable exception from this picture is again $\mathrm{I}_{6}$. Rate fluctuation is the only factor determining its covariation with $I_{1}$ to $I_{5}$, according to the parallel model, and these measures provide direct estimates of $\sigma_{X}^{2}$ for this reason.

Variances of $I_{1}$ to $I_{6}$. The variances for $I_{1}$ to $I_{6}$ were derived by the same method as the covariances by computing the covariances of the terms in Table 3 with themselves. Note that the effects of timekeeper variability are not included in Table 4. For simplification, we assume that rate fluctuation and timekeeper variability play a negligible role for those tempos at which parallel timing can be reasonably expected and focus on the role of the asynchrony at this point: The variances of intervals $I_{0}$ to $I_{5}$ depend on $\sigma_{0}^{2}$. In contrast, $I_{6}$ should not be affected by $\sigma_{0}^{2}$. Instead, the variance of $I_{6}$ permits a direct test of our assumption that rate and timekeeper variance play a negligible role at rapid tempos.

\section{Summary and Contrast of Model Predictions}

Table 5 lists the sources of covariation for the integrated and the parallel models. For a broader theoretical perspective, we included the Wing-Kristofferson model and the extended two-level timing framework.

All four models are compatible with the assumption that variances of observed intervals increase with realized duration. The two-level timing assumption is also incorporated in all four models, that is, the prediction that shared motor delays induce negative covariation between adjacent intervals. The original formulation of the Wing-Kristofferson model is silent with respect to asynchronies in bimanual performance. It can be integrated into the extended twolevel timing framework by assuming that the same clock controls both hands. Asynchronies in simultaneous keystrokes are attributed to differences in motor delays in this framework. This extension was proposed and tested by Vorberg and Hambuch (1984). In this respect, the integrated model makes the same predictions as the extended two-level timing framework. The integrated and the parallel models distinguish between covariation introduced by rate fluctuation during the programming stage and variance during timekeeper execution (rhythm program hypothesis). From this perspective, the Wing-Kristofferson model corresponds to the special case in which (a) rate fluctuation is zero and (b) all successive intervals are controlled by the same reprogrammable clock. Different from that, we predict that rate fluctuation has a marked effect at slower tempos and that it increases with cycle duration. Both the integrated and the parallel models predict that rate fluctuation causes positive covariation between intervals $I_{1}$ to $I_{6}$, the magnitude of which depends on the mean interval durations. Variances should also reflect the proportional effects of rate fluctuation; in addition, the effects of timekeeper execution should lead to an even stronger increase with interval duration of variances as compared with covariances.

The critical differences between the integrated and the parallel models relate to the role of the asynchrony. With the 
Table 4

Covariance Matrix Predicted From the Parallel Model for the Polyrhythmic Task

\begin{tabular}{|c|c|c|c|c|c|c|}
\hline Interval (I) & $I_{1}$ & $\mathbf{I}_{2}$ & $I_{3}$ & $\mathbf{L}_{4}$ & $I_{5}$ & $\mathbf{I}_{6}$ \\
\hline$L_{0}$ & $-\sigma_{0}^{2}-m_{L}$ & $\sigma_{0}^{2}$ & $-\sigma_{0}^{2}$ & $\sigma_{0}^{2}$ & $-\sigma_{0}^{2}$ & 0 \\
\hline$I_{1}$ & $\begin{array}{l}\sigma_{0}^{2}+\left(\mu_{1}\right)^{2} \\
\quad \sigma_{X}^{2}+m_{R}+m_{L}\end{array}$ & $\begin{array}{c}-\sigma_{0}^{2}+\mu_{1} \mu_{2} \\
\sigma_{X}^{2}-m_{R}\end{array}$ & $\sigma_{0}^{2}+\mu_{1} \mu_{3} \sigma_{X}^{2}$ & $-\sigma_{0}^{2}+\mu_{1} \mu_{4} \sigma_{X}^{2}$ & $\sigma_{0}^{2}+\mu_{1} \mu_{5} \sigma_{X}^{2}$ & $\mu_{1} \mu_{6} \sigma_{x}^{2}$ \\
\hline $\mathrm{I}_{2}$ & & $\begin{array}{l}\sigma_{0}^{2}+\left(\mu_{2}\right)^{2} \\
\quad \sigma_{X}^{2}+m_{R}+m_{L}\end{array}$ & $\begin{array}{c}-\sigma_{0}^{2}+\mu_{2} \mu_{3} \\
\sigma_{X}^{2}-m_{L}\end{array}$ & $\sigma_{0}^{2}+\mu_{2} \mu_{4} \sigma_{X}^{2}$ & $-\sigma_{0}^{2}+\mu_{2} \mu_{5} \sigma_{X}^{2}$ & $\mu_{2} \mu_{6} \sigma_{X}^{2}$ \\
\hline$I_{3}$ & & & $\begin{array}{l}\sigma_{0}^{2}+\left(\mu_{3}\right)^{2} \\
\quad \sigma_{X}^{2}+m_{R}+m_{L}\end{array}$ & $\begin{array}{c}-\sigma_{0}^{2}+\mu_{3} \mu_{4} \\
\sigma_{X}^{2}-m_{R}\end{array}$ & $\sigma_{0}^{2}+\mu_{3} \mu_{5} \sigma_{X}^{2}$ & $\mu_{3} \mu_{6} \sigma_{X}^{2}$ \\
\hline $\mathbf{I}_{4}$ & & & & $\begin{array}{l}\sigma_{0}^{2}+\left(\mu_{4}\right)^{2} \\
\quad \sigma_{X}^{2}+m_{R}+m_{L}\end{array}$ & $\begin{array}{c}-\sigma_{0}^{2}+\mu_{4} \mu_{5} \\
\sigma_{X}^{2}-m_{L}\end{array}$ & $\mu_{4} \mu_{6} \sigma_{X}^{2}$ \\
\hline $\mathrm{I}_{5}$ & & & & & $\begin{array}{l}\sigma_{0}^{2}+\left(\mu_{s}\right)^{2} \\
\quad \sigma_{X}^{2}+m_{R}+m_{L}\end{array}$ & $\mu_{5} \mu_{6} \sigma_{X}^{2}-m_{R}$ \\
\hline $\mathbf{I}_{6}$ & & & & & & $\left(\mu_{6}\right)^{2} \sigma_{X}^{2}+2 m_{R}$ \\
\hline
\end{tabular}

Note. $\quad \sigma_{0}^{2}=$ Variance of the asynchrony $I_{0} ; \sigma_{X}^{2}=$ rate fluctuation (variance of $X$ ); $\sigma_{X}^{2}=q_{0}+q_{1} E(X)+q_{2} E^{2}(X) ; \mu=$ sums of proportions of $X$ realized by timekeepers for right and left hands, respectively; $\mu_{1}=a, \mu_{2}=e-a, \mu_{3}=a+b-e, \mu_{4}=e+f-a-b, \mu_{5}=a+b+c-$ $e-f, \mu_{6}=\mathrm{d} ; m_{R}$ and $m_{L}=$ variance of motor delays for right and left hands, respectively.

exception of adjacent intervals, the integrated model predicts only zero or positive covariances. The checkerboard pattern predicted by the parallel model thus provides a valuable indicator of distinguishing integrated and parallel timing control. A successful demonstration of the negative covariances in the checkerboard pattern presumes that rate fluctuation and timekeeper variability are small relative to the variance of the asynchrony, or else the positive covariances will cancel out negative covariation. We maintain that this is a plausible assumption for rapid tempos, and we argued earlier that the benefits of parallel timing are limited to those fast rates in the first place.
So how can we distinguish integrated and parallel models for those, presumably slow, tempos in which the effects of rate fluctuation and timekeeper variability are large compared with the variance of the asynchrony? A related question is, How can we ascertain that participants do not rely on parallel timing all along? A direct test for our hypothesis that integrated timing is the control mode for slower tempos can be conducted on the variances of within-hands intervals. Consider the four right within-hands intervals in the polyrhythmic task (see the bottom panel in Figure 2): The parallel model assumes that within-hands intervals $R_{1}$ to $R_{4}$ are directly controlled by one single

Table 5

Sounces of Covariation and Their Predicted Effects in Four Different Models

\begin{tabular}{|c|c|c|c|c|c|}
\hline \multirow[b]{3}{*}{$\begin{array}{c}\text { Model } \\
\text { (interval [I] timed) }\end{array}$} & \multicolumn{5}{|c|}{ Criterion measure } \\
\hline & \multicolumn{2}{|c|}{ Asynchrony and covariances with $I_{1}$ to $I_{6}$} & \multicolumn{2}{|c|}{ Covariances between $I_{1}$ to $I_{6}$} & \multirow[b]{2}{*}{$\begin{array}{l}\text { Variances } \\
\text { of } I_{1} \text { to } I_{6}\end{array}$} \\
\hline & $\begin{array}{l}\text { Asynchrony } \\
\text { variance }\end{array}$ & $\begin{array}{l}\text { Covariance } \\
\text { with } I_{1} \text { to } I_{6}\end{array}$ & Adjacent & $\begin{array}{c}\text { Non- } \\
\text { adjacent }\end{array}$ & \\
\hline $\begin{array}{l}\text { Wing \& Kristofferson } \\
\text { (successive, nonover- } \\
\text { lapping) }\end{array}$ & & & Motor delays (-) & 0 & $\begin{array}{l}\text { Clock } \\
\text { Motor delays }\end{array}$ \\
\hline $\begin{array}{l}\text { Extended two-level timing } \\
\text { (successive, nonover- } \\
\text { lapping) }\end{array}$ & Motor delays & $\frac{0}{I_{1}: \text { Motor delays (-) }}$ & Motor delays ( - ) & 0 & $\begin{array}{l}\text { Timekeeper execution } \\
\text { Motor delays }\end{array}$ \\
\hline $\begin{array}{l}\text { Integrated timing (succes- } \\
\text { sive, nonoverlapping) }\end{array}$ & Motor delays & $\begin{array}{c}0 \\
\mathbf{I}_{1}: \text { Motor delays }(-)\end{array}$ & $\begin{array}{l}\text { Rate }(+) \\
\text { Motor delays (-) }\end{array}$ & Rate (+) & $\begin{array}{l}\text { Rate } \\
\text { Timekeeper execution } \\
\text { Motor delays }\end{array}$ \\
\hline \multirow{2}{*}{$\begin{array}{l}\text { Parallel timing (within- } \\
\text { hands, overlapping) }\end{array}$} & Error correction & \multicolumn{3}{|c|}{$I_{1}$ to $I_{5}$ : Asynchrony (checkerboard $+/-$ ); Exception: $I_{6}$} & Asynchrony $\left(I_{1}\right.$ to $\left.I_{5}\right)$ \\
\hline & & & $\begin{array}{l}\text { Rate }(+) \\
\text { Motor delays }(-)\end{array}$ & Rate $(+)$ & $\begin{array}{l}\text { Rate } \\
\text { Timekeeper execution } \\
\text { Motor delays }\end{array}$ \\
\hline
\end{tabular}

Note. (+) and (-) denote positive and negative covariation, respectively. All effects are positive in the two variance columns. 
timekeeper each; in the integrated model, intervals $\mathbf{R}_{\mathbf{2}}$ and $\mathbf{R}_{\mathbf{3}}$ emerge from concatenation of timekeeper signals for between-hands intervals. The integrated model predicts higher variances for $R_{1}$ and $R_{4}$ compared with the concatenated intervals $R_{2}$ to $R_{3}$ because of the quadratic terms in our equations for timekeeper execution variance. As an example, for perfect realization at the level of mean intervals (i.e., $\left.\mathbf{R}_{1}=\mathbf{R}_{4}=1 / 4 X ; \mathbf{R}_{2}=\mathbf{R}_{3}=1 / 12 X+1 / 6 X\right)$, the expected mean durations are identical; however, the timekeeper variance must differ because $(1 / 4)^{2}>(1 / 12)^{2}+(1 / 6)^{2}$. In contrast, the parallel model predicts identical variances for $R_{1}$ to $R_{4}$.

\section{Method}

\section{Participants}

Fifteen expert pianists, all master students from two Berlin music academies, were recruited for the study. All of them had already performed in public concerts and were working toward careers as performing artists. One person could not complete the experiment because of concert commitments abroad, leaving 14 participants ( 7 women and $7 \mathrm{men}$ ) for final analysis. The pianists came from a variety of cultural and ethnic backgrounds, including Japan $(n=4)$, Israel $(n=1)$, Russia $(n=1)$, and Germany $(n=8)$, reflecting the reputation and standards of the two academies and the high selection criteria for our sample. The pianists' mean age was 23.93 years $(S D=2.64$, range $=20-30)$. On average, they had started piano instruction at the age of 5.86 years $(S D=2.18)$ and had received 18.39 years of formal instruction $(S D=3.55)$ since then. All but one reported to be right-handed. They were paid $300 \mathrm{DM}$ for their participation.

\section{Procedure}

Participants performed all experimental tasks on a Yamaha CLP-124 piano with a weighted keyboard mechanic connected to a Macintosh II computer through a MIDI interface. The controls of the piano were set to a comfortable volume, such that participants could monitor their performance throughout the entire trial. Onsets and offsets of keystrokes were assessed to the nearest millisecond. Data were collected over the course of seven sessions, with each lasting between 1.5 and $2 \mathrm{hr}$. In Session 1 we obtained biographical information and retrospective estimates of past amounts of deliberate practice. Participants then performed three simple fingertapping tasks (right, left, and alternate index fingers); these were repeated at the end of Session 1 . We then assessed tapping speed in all three conditions during each of Sessions $2-6$ at the beginning and at the end of each session. Participants performed a musical interpretation task at the beginning of Session 3. The focus of this article is on the rhythm production tasks.

In each session only one of the two tasks was administered. We alternated tasks between sessions beginning with the control task (syncopated rhythm) in Session 1. During each of Sessions 1-6, participants performed two blocks of trials, one for each rhythm task; within each block, 14 different metronome tempos ranging from 800 to $8,200 \mathrm{~ms}$ per cycle were administered in a randomized order. Error trials or trials with more than $10 \%$ deviation from the target timing in either hand were repeated up to a maximum of five trials for a certain task-tempo condition. In Session 7 we assessed performance limits with respect to speed. To this end, we administered blocks with a fixed number of speeded trials and gradually increased the tempo of successive blocks in 100-ms steps. Participants started with a cycle duration of $800 \mathrm{~ms}$ and were administered a maximum of 12 trials per tempo if he or she was capable of performing the task at a certain tempo. We proceeded to the next faster rate if a participant produced at least 6 valid trials at a given tempo.

The overall procedure followed the continuation paradigm (Wing \& Kristofferson, 1973a). At the beginning of each trial, the computer generated the exact rhythmic pulse as three different sinusoidal tones. In pilot work with a trained pianist, we tried to find pacing tones that were maximally distinct while still sounding acceptable in the context of the polyrhythmic task. We chose the following combination of tones: The beginnings of each cycle were marked by a middle $\mathrm{C}(261-\mathrm{Hz}, 50-\mathrm{ms}$ duration), and within-cycles left-hand strokes were indicated by a $\mathrm{C}$ one octave above the first tone (523- $\mathrm{Hz}, 30-\mathrm{ms}$ duration). To enhance distinction of the right- and left-hand tones, within-cycles right-hand strokes were an octave above the left-hand tone $(1046-\mathrm{Hz}, 30-\mathrm{ms}$ duration). We created envelopes with similar slopes for the tones to prevent clicks or unpleasant bursts.

Participants played with their index fingers on two white keys of the digital piano. The two target keys were $\mathrm{C} 3$ and $\mathrm{C} 4$ (i.e., the right hand playing one octave above the left). The volume of the digital piano was set such that pianists could comfortably monitor their performance. At the onset of each trial, they listened to the pacing pulse as long as they wanted. After they started, pianists played along with the computer beat for four full cycles (the synchronization phase), after which the pulse was discontinued and data recording commenced. Participants continued for an additional 12 cycles without the external support (the continuation phase). Only these data were entered into later analyses. After each trial, participants received error feedback or (for correct trials) detailed graphic feedback on their performances. The feedback consisted of three lines of column diagrams illustrating the realized duration (top line) of cycles as well as the timing of single intervals. Target durations were indicated by a reference line for each type of interval. To make the feedback as informative as possible and to encourage parallel timing at the same time, we used different formats for the feedback on single intervals in the two tasks: In the control condition with its isochronous structure, groups consisting of the six successive, nonoverlapping intervals per cycle were displayed across Lines 2 and 3 . In the polyrhythm task 12 groups of four right-hand intervals each were displayed on Line 2, and the corresponding 12 groups of three left-hand intervals each were shown in Line 3 . In addition, values for percentage deviations from the prescribed durations were displayed for cycle means, right hand, and left hand, respectively. This summary information indicated to the participant whether a trial had to be repeated, which was the case if any of the three deviation values exceeded $10 \%$.

\section{Results}

We first provide some general information about errors and data screening. We then present a qualitative illustration for our major claims: (a) At slower tempos the data suggest an integrated organization in which concatenated timekeepers control both hands and (b) at rapid tempos the pattern of covariances implies a parallel control mode. We next fit the integrated model to the data from slower tempos and focus on the evidence for the claim that integrated timing cannot account for the pattern of covariances emerging in rapid polyrhythmic performance. We show that our simplified version of a parallel model is qualitatively in good agreement with the data. We conclude our discussion of results with a contrast of integrated and parallel timing of withinhands intervals that highlights the differential benefits of both control modes at different tempos. 


\section{Errors and Initial Data Screening}

A total of 3,778 trials was collected in the course of the seven-session experiment; 1,781 in the syncopated rhythm task and 1,997 in the polyrhythmic condition. Besides excluding trials with errors in the prescribed sequence of keystrokes, we applied three criteria at the level of individual trials in our data screening. These criteria related to (a) mean realized tempo, (b) single interval outliers, and (c) extreme values for the variability of mean realized cycle durations. In the analyses that follow, we distinguish between "fast" (i.e., 1,200 ms/cycle and below) and "slow" (i.e., $1,400 \mathrm{~ms} /$ cycle and above) tempos for reasons that we detail in the next section.

Not all participants provided acceptable trials at rapid tempos. To avoid unequal numbers of participants across cells, we limited our analyses of fast tempos to those 6 participants who mastered the $600-\mathrm{ms} /$ cycle conditions in both tasks. Our selected 6 participants produced a total of 753 trials (391 in the polyrhythmic task and 362 in the syncopation control task) at tempos $1,200 \mathrm{~ms} /$ cycle and faster. After screening, the mean numbers of trials at rapid rates provided by our selected 6 participants were $8.5,9.8$, $13.7,7.0$, and 8.2 for cycle durations of $600,700,800,1,000$, and $1,200 \mathrm{~ms} / \mathrm{cycle}$ in the polyrhythmic task. The large number of trials in the 800 -ms condition is due to the fact that this tempo was the only one assessed in Sessions 1-6 as well as Session 7 for all participants. The corresponding numbers for the syncopated rhythm task were $6.7,7.6,16.3$, 6.7, and 5.7. Note that 5 of these 6 pianists also provided a limited number of acceptable trials in the $500-\mathrm{ms} / \mathrm{cycle}$ polyrhythmic condition. None of our participants could do so in the syncopated rhythm task.

The data from all 14 participants (a total of 2,055 trials; 1,050 in the polyrhythmic task and 1,005 in the syncopated rhythm task) formed our initial database for slow tempos (cycle durations of 1,400-8,200 ms). For slow tempos, the final number of trials per participant and metronome cycle duration surviving our data screening was 5.91 on average in the polyrhythmic task and 5.70 trials in the syncopated rhythm task.

Sequence errors. We considered a cycle as correct if participants pressed the right and left keys in the prescribed order and if both keys were in the down position at the same time for the simultaneous keystroke defining the beginning of each cycle. Only trials in which all 12 continuation cycles were correct according to this criterion were included in the analysis. On average, participants had to repeat 2.89 trials per task (summed across all 11 slow tempo conditions), and there were no differences between rhythmic tasks in that respect. This criterion led to an exclusion of $3.94 \%$ of the trials collected at slower tempos.

Deviations from metronome tempo. To screen out trials that violated the prescribed tempo, we defined symmetric, nonoverlapping target windows around the ideal cycle durations. As an example, for metronome tempo conditions of $2,000,2,400$, and $2,800 \mathrm{~ms} /$ cycle, we excluded trials with a mean realized cycle duration that deviated by more than $200 \mathrm{~ms}$ from the ideal value. Across all slow tempos, this was the case for 6.57 trials per participant and task, leading to an exclusion of $8.95 \%$ of the slow trials. We found no differences between tasks in this respect. The number of trials affected increased significantly with cycle duration, suggesting that participants had more difficulties to properly match and maintain slow tempos. In the case of the extremely rapid tempos $(500-700 \mathrm{~ms} / \mathrm{cycle})$ we applied a more lenient criterion; we included all trials with a mean tempo up to $70 \mathrm{~ms}$ slower than the ideal value.

Outliers. We screened our data for those trials in which any of the seven observed intervals (see Figure 2) deviated by more than $3 S D$ s from the mean computed for this interval in a given trial. This criterion rarely applied after the earlier screening and concerned only 8 trials altogether. Finally, we identified trials with extreme values for the standard deviation of realized cycle durations applying the interquartile-stretch criterion (Hoaglin, Mosteller, \& Tukey, 1983) on all trials remaining for each task and tempo condition. For slow tempos, this criterion affected 1.71 trials per participant in the syncopation task and 2.57 trials in the polyrhythmic condition. In total, we excluded another $1.46 \%$ of the slow trials initially collected for this reason.

\section{Asynchrony and Its Covariances With the Remaining Intervals: Overall Pattern}

Figure 3 shows the covariance pattern related to $I_{0}$ for both tasks across the whole tempo range assessed. ${ }^{2}$ The right part of either figure depicts tempos of 1,400-ms cycle

2 We used the estimator for covariances described in Vorberg and Hambuch (1978). For two intervals $I_{j}$ and $I_{k}$, their covariance is estimated by

$$
c_{j k}(m)=\frac{\sum_{i=1}^{n-m} \mathrm{I}_{i j} \mathrm{I}_{i}+{ }_{m, k}-\left(\frac{1}{n-m}\right) \sum_{i=1}^{n-m} \mathrm{I}_{i j} \sum_{i=1}^{n-m} \mathrm{I}_{i}+\mathrm{I}_{i}+{ }_{m, k}}{n-m-1},
$$

where $n$ denotes the number of cycles in a series (i.e., the number of cycles in each trial) and $m$ is the cycle lag. For the most part, we present covariances between intervals from the same cycle that is for $m=0$. For convenience, we omit the cycle index and denote these covariances as $c_{j k}$. For example, the covariance between adjacent intervals $I_{1}$ and $I_{2}$ is denoted as $c_{12}$; because the variance equals the covariance of a variable with itself, we denote the variance of $I_{l}$, for example, as $c_{11}$. We limit our considerations of covariances across cycles to those involving the asynchrony at cycle-lag $1(m=1)$; we denote those covariances as $c$. For example, the covariance between the last interval $I_{6}$ and the asynchrony in the next cycle is referred to as $\mathrm{c} 6 \mathrm{6}$. As Vorberg and Hambuch (1978) have shown, covariance estimates are unbiased for $m=0$. This is not true if covariances are computed across cycles or separated by cycle boundaries, which is inevitably the case if the first $\left(I_{0}\right)$ and the last $\left(\mathrm{I}_{6}\right)$ interval are correlated. We applied the following bias correction for the two covariances in question:

$$
\begin{gathered}
G_{06}(0)=E_{06}(0)+2 / n E_{06}(1) \\
G_{06}(1)=E_{06}(1)+1 /(n-1) E_{06}(0),
\end{gathered}
$$



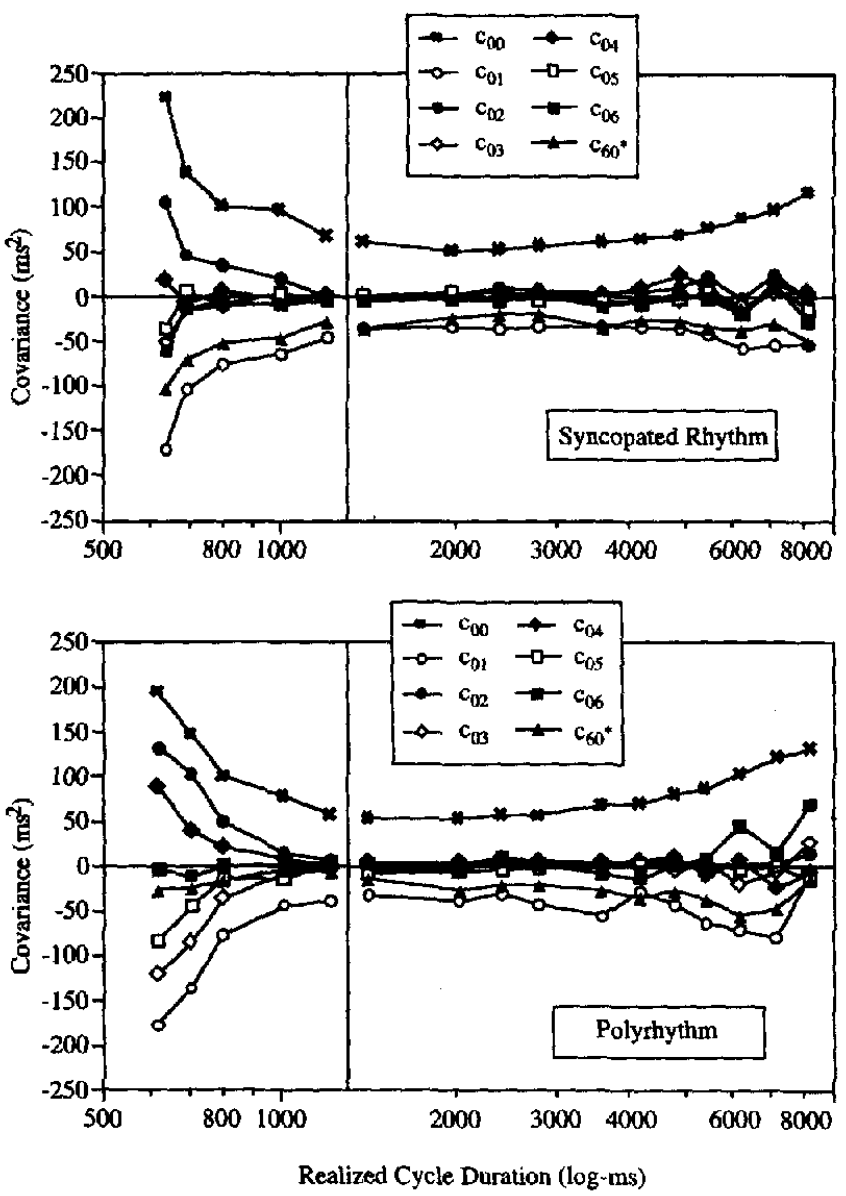

Figure 3. Covariances between asynchrony $\left(\mathrm{I}_{0}\right)$ and the remaining intervals as a function of realized cycle duration. The top panel shows data from the syncopated rhythm task; the bottom panel shows data from participants' polyrhythmic performance. The values in the left part of each panel are averaged from the 6 participants showing the best performances at rapid tempos. All 14 participants contributed to the data points at slower tempos depicted in the right sections of each panel. $c_{00}=$ the variance of the asynchrony $\mathrm{I}_{0} ; \mathrm{c}_{01}$ to $\mathrm{c}_{06}=$ the covariances of the asynchrony with the remaining intervals later in the cycle; $\mathrm{c}_{0_{0}}=$ the covariance of the last interval in the cycle $\left(\mathrm{I}_{6}\right)$ with the asynchrony in the following cycle.

durations and above. The predictions of the extended two-level timing framework (and the integrated model) appear to be met under these conditions: Covariances reflecting left-hand $\left(c_{01}\right)$ and right-hand $\left(c_{6_{0}}\right)$ motor delays were negative; all other covariances were close to zero. The variance of $I_{0}$ (denoted by $c_{00}$ in Figure 3) was close to the sum of the absolute values of the two adjacent covariances. A different picture emerged for the "fast side," that is, for tempos $1,200 \mathrm{~ms}$ per cycle and below. First, the variance of

where $E$ is the estimate according to the above formula; $G$ is the bias-corrected estimate. In the text and all figures, we report the bias-corrected values, where $G_{06}(0)$ corresponds to $c_{06}$ and $G_{06}(1)$ corresponds to $\mathrm{c}$.
$I_{0}$ increased with tempo. Second, the covariances between the asynchrony and the other intervals in the cycle showed the pattern of alternating signs predicted by the parallel model.

In the next section, we fit the integrated model to the data from slower tempos. First, we confirm the impression from Figure 3 that the extended two-level timing framework can account for the asynchrony and its covariances on the "slow" side of the performance function by quantitative modeling. We then extend the scope of our model testing to the complete covariance matrix to evaluate the integrated model's predictions concerning the effects of rate fluctuation and timekeeper execution. We return to the "fast side" later.

\section{Timing at Slower Tempos: The Integrated Model}

Our analyses of performance at slower tempos (i.e., 1,400 $\mathrm{ms} / \mathrm{cycle}$ and above) were guided by three questions: (a) whether the extended two-level timing framework provides a sufficient account for our data if the complete covariance matrix is considered; (b) whether systematic violations of the extended two-level timing framework's predictions support the assumption of additional processes; and (c) whether we can identify a model that can account for both the performance in the polyrhythmic and the syncopated rhythm task. All model fitting was done with the program for canonical nonlinear regression (CNLR) in SPSS-X. We turn first to the asynchrony and its covariances with the other intervals.

Asynchrony and covariances with $I_{1}$ to $I_{6}$ : Motor delays. As a first step we compared the two covariances, $c_{01}$ reflecting left-hand and $c w_{0}$ reflecting right-hand motor delay variances across tasks. We conducted a repeated measures analysis of variance (ANOVA) on $c_{01}$ and $c_{60}$ with hand, task, and tempo (11 cycle durations ranging from 1,400 to $8,200 \mathrm{~ms} /$ cycle) as within-subjects factors. The main effect of hand was significant, $F(1,13)=6.50, M S E=2,993, p<$ .05 , indicating that on average the motor delay variance for the left hand was larger $\left(M=42.91 \mathrm{~ms}^{2}, S D=13.40\right)$ than the right-hand variance $\left(M=30.10 \mathrm{~ms}^{2}, S D=19.18\right)$. This effect shows up in Figure 3 as more negative values for $\mathfrak{c}_{01}$ than $\mathrm{c}_{\delta_{0}}$ in both tasks. A polynomial contrast was used for the tempo factor. The linear trend was significant, $F(1,13)=$ $4.93, M S E=5,346, p<.05$, because of the increase in the absolute magnitude of covariances at longer durations. None of the other main effects or interactions including task $(p>.25$ ) was significant.

Subsequently, we fitted a second-order polynomial to the data of each individual. The dependent measures were $c_{01}$, $\mathrm{c}_{60}^{*}$, and $\mathrm{c}_{00}$ assessed at 11 different tempos. Within each tempo, we averaged each of the three measures across tasks, obtaining 33 empirical cells per participant. To account for the mean differences between right- and left-hand motor delay variances evident from the ANOVA, we permitted two different intercepts for right and left hands, respectively. Thus, four parameters were estimated for each participant. Figure 4 shows the observed values (a subset of the data in 


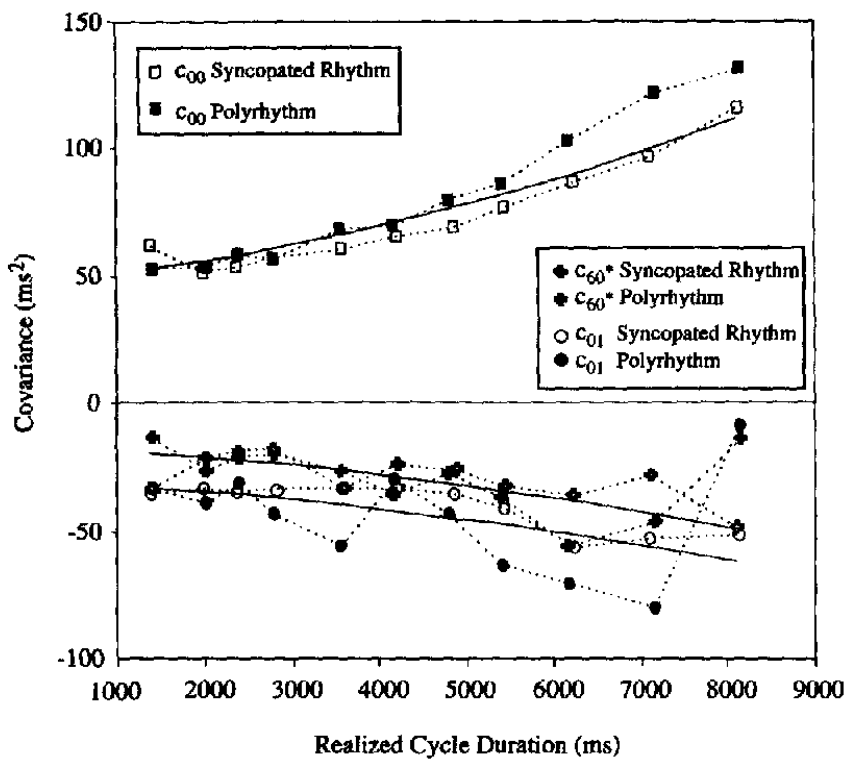

Figure 4. Predicted (solid lines) and observed variances of the asynchronies (interval $I_{0}$ ) and covariances between $I_{0}$ and adjacent intervals reflecting left-hand $\left(c_{01}\right)$ and right-hand $\left(c_{\sigma_{0}}\right)$ motor delays as a function of realized cycle durations. Individual model estimates were obtained for a tempo range of $1,400-8,200 \mathrm{~ms} / \mathrm{cycle}$ from covariances averaged across tasks. The curve fits are based on averaged values for individual model estimates.

the right parts of Figure 3) and curve fits from averaged individual estimates.

The averaged model estimate for the variance of the asynchrony was $74.85 \mathrm{~ms}^{2}(S D=25.15)$, that is, close to the empirical value, which was $75.84 \mathrm{~ms}^{2}(S D=25.24)$. Consistent with the model predictions, both values were in the range of to the mean sum of the absolute values of $c_{01}$ and c 60, which was $73.01 \mathrm{~ms}^{2}$. Averaged model estimates for delay variances were $31.09 \mathrm{~ms}^{2}(S D=17.22)$ for the right hand and $43.77 \mathrm{~ms}^{2}(S D=12.35)$ for the left hand. Both values were close to the means of the observed values reported earlier. Thus, the predictions of the extended two-level timing framework for covariances including the asynchrony are in line with our data, with the qualification that motor delay variances tended to increase with duration.

According to Wing and Kristofferson's (1973a) model, all covariances between intervals have zero (nonadjacent) or negative (adjacent) predictions. The integrated model is a generalized variant of the extended two-level timing framework in that it predicts positive covariation between intervals $I_{1}$ to $I_{6}$ due to rate fluctuation. We now turn to these covariances and their dependence on tempo.

Covariances between $I_{1}$ to $I_{6}:$ Rate fuctuation. Figure 5 illustrates a selection of five covariances between nonadjacent interval pairs. The curve fits represent averaged individual estimates from our fit of the integrated model. We describe the details of the fit procedure below. The data show that the extended two-level timing framework cannot account for the pattern of results without assuming additional processes: The covariances were positive and rose systemati- cally with cycle duration. The covariance matrix for each task and tempo condition contained 10 cells for nonadjacent pairs of intervals $I_{1}$ to $I_{6}$ (see Table 1). Only 2 of the 220 values obtained from the two tasks and 11 tempo conditions were negative $(-4$ and -7$)$. In contrast, covariances between adjacent pairs of intervals $I_{1}$ to $I_{6}$ were mostly negative at tempos of 1,400 to $2,400 \mathrm{~ms} /$ cycle $(26$ of 30 pairs) with respective correlations perfectly in the 0 to -.5 range predicted by the extended two-level timing framework. ${ }^{3}$ At slower tempos these covariances became increasingly more positive; for tempos of $3,600 \mathrm{~ms} /$ cycle and above, all covariances were positive except for those that include the asynchrony. The covariances in the syncopated rhythm task, with its isochronous structure, assume similar values, and this was equally true for those pairs not shown in Figure 5. At the same time, the magnitudes of covariances in the polyrhythmic task varied with the average duration of the intervals as predicted by the integrated model.

The relation between overall tempo and rate fluctuation is described by the second-order polynomial in Equation 5 (theory section). The proportional factors in the integrated model's predictions for the covariances between intervals (see Table 1) can be calculated from the mean realized intervals and the mean cycle duration as described earlier. The parameters $\left(q_{0}, q_{1}\right.$, and $\left.q_{2}\right)$ were estimated from the data. To compare the effects of rate fluctuation between the two tasks, we first estimated the three parameters separately for the polyrhythmic and the syncopation tasks. Model fitting was done at the level of individual participants. To avoid a bias of the minimum least square loss function toward large covariances (i.e., large interval pairs at slow tempos), we fitted correlations. To this end, the 21 cells in Table 1 were standardized by the observed variances for each task and tempo condition. Initial runs suggested that convergence was greatly improved if the slowest tempo $(8,200 \mathrm{~ms} / \mathrm{cycle})$ was excluded, leaving $210(21 \times 10$ tempos $)$ empirical cells for the estimation of three parameters in each task condition. We used the estimates for the motor delay variances in our equations for covariances between adjacent intervals.

Subsequently, we ran a repeated measures ANOVA on the rates estimated by this procedure, with task (control vs. polyrhythm) and tempo (10 cycle durations) as withinsubjects factors. Not surprisingly, both linear, $F(1,13)=$ $79.89, M S E=30,077,357, p<.001$, and quadratic trends, $F(1,13)=97.98, M S E=3,766,258, p<.001$, were significant. More important, neither the main effect of task nor the interactions between task and tempo approached significance $(p>3)$. We may thus assume similar effects of rate fluctuation in both tasks. Based on these findings, we estimated the rate parameters again, fitting one set of parameters to the data from both tasks. The curves in Figure 5 show these estimates averaged across participants.

\footnotetext{
${ }^{3}$ Distributivity does not hold for correlations, which is a problem for averaging correlations from single trials or across participants. All correlations reported in this article were computed by standardizing the average covariances by the average variances at the level reported in the text.
} 

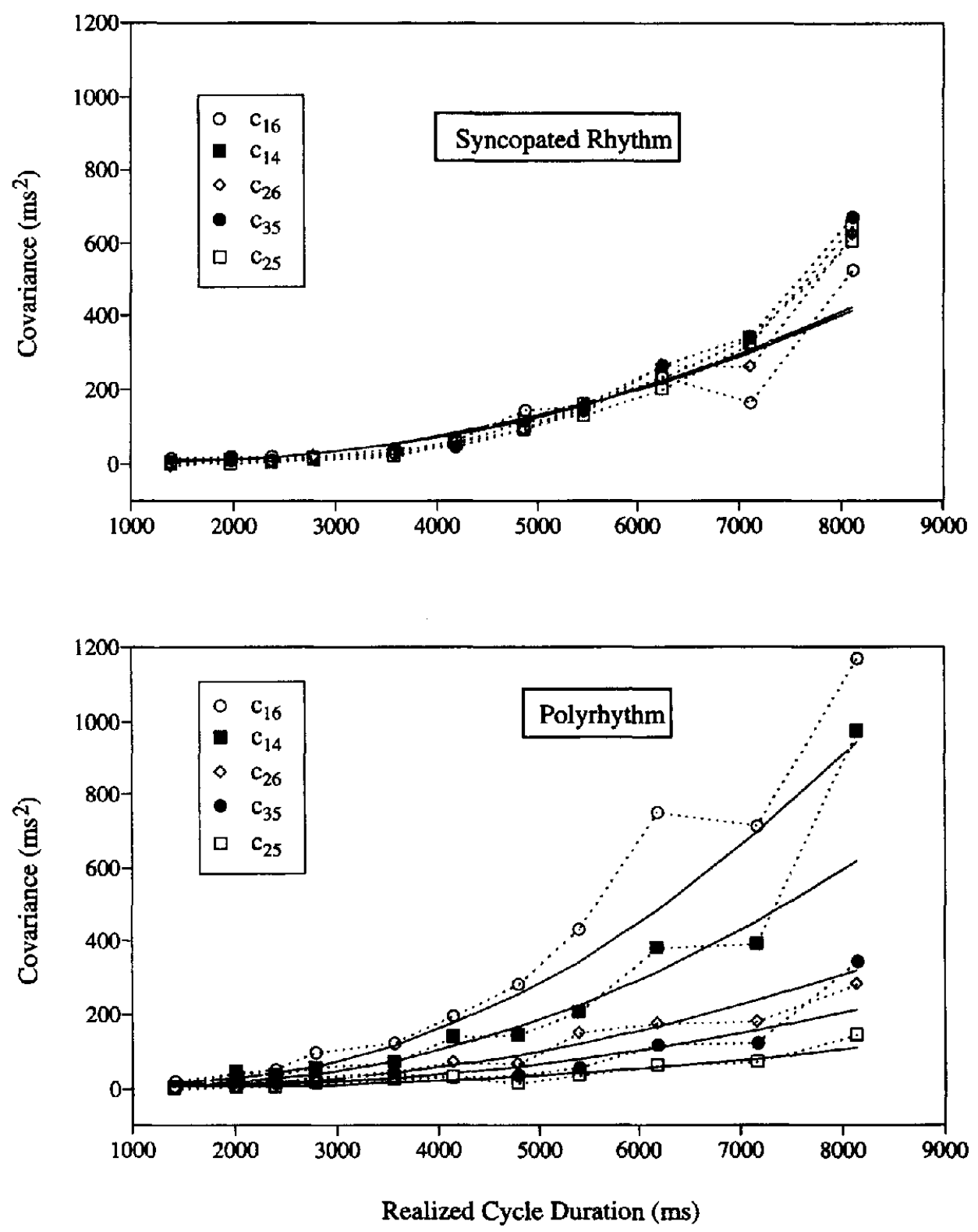

Figure 5. Observed covariances between nonadjacent intervals and the rate fluctuation model's predictions (solid lines) at slow tempos as a function of realized cycle durations for the syncopated rhythm with isochronous structure (top panel) and the polyrhythmic task (bottom panel). Interval pairs were chosen to reflect different proportions in the polyrhythmic task. For example, $c_{16}$ is the covariance between intervals $I_{1}$ and $I_{6}$, the two long intervals in the polyrhythmic task. The covariance between the two shortest intervals in the same task is $c_{25}$. Model parameters were estimated simultaneously for both tasks for a tempo range of $1,400-8,200 \mathrm{~ms} / \mathrm{cycle}$ for each participant. The curve fits are based on averaged values for individual model estimates.

Variances of $I_{1}$ to $I_{6}$ : Timekeeper execution. The assumption of rate fluctuation explains strikingly different patterns of covariances between intervals in both tasks. Figure 6 shows the observed variances for the syncopated rhythm task (top panel) and the polyrhythmic task (bottom panel) along with the integrated model's predictions averaged across participants. The integrated model is based on the assumption that the variances reflect the summed contribution of rate fluctuation during the programming stage and timekeeper variance during execution. For a direct illustration of the dissociability of the two processes in the data, consider the syncopated rhythm task, in which intervals $I_{1}$ to $I_{6}$ have the same (ideal) proportions $(1 / 6 X)$. If the effects of rate fluctuation during target specification were the only 

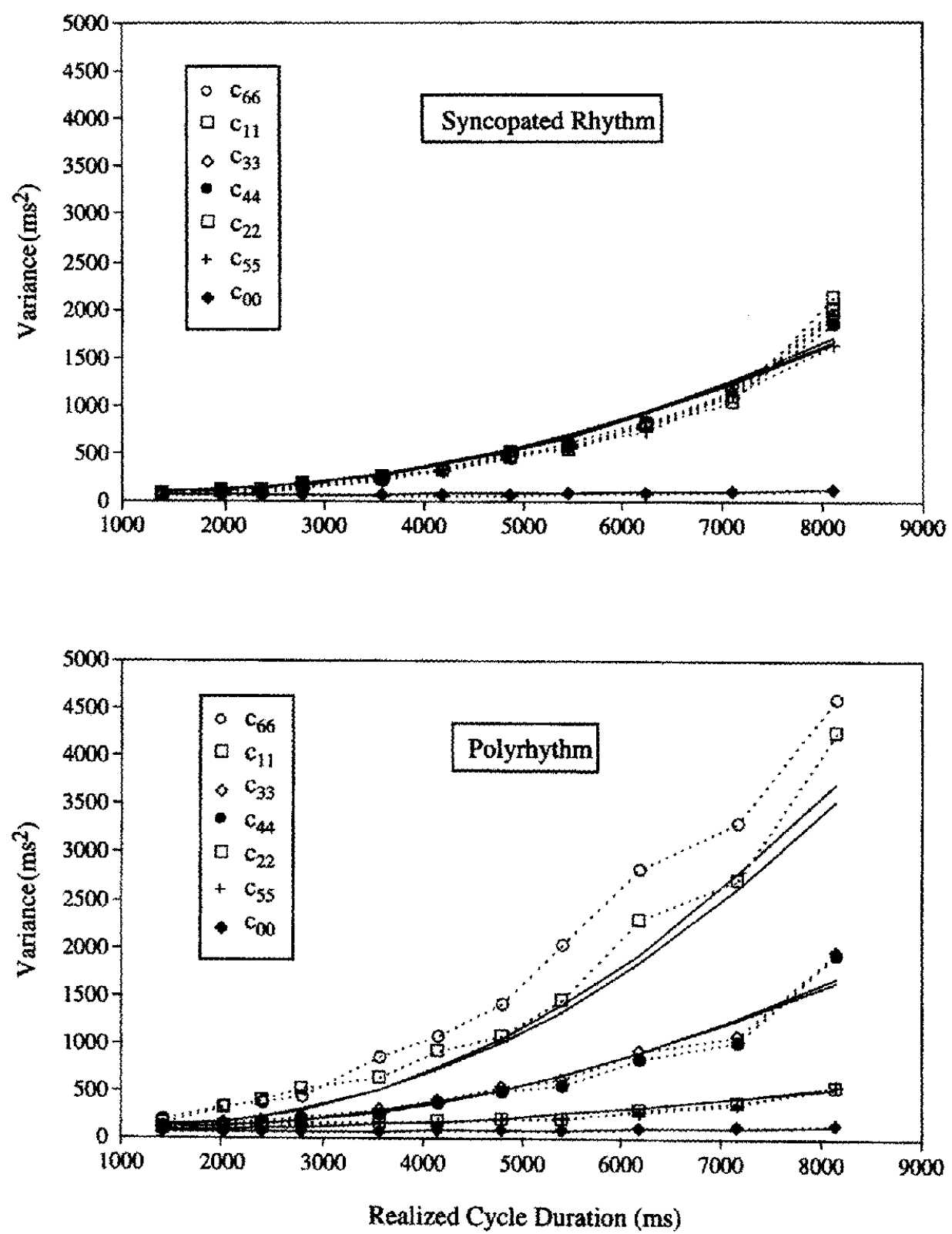

Figure 6. Estimated and observed variances at slow tempos as a function of realized cycle durations for the syncopated rhythm task with isochronous between-hands intervals (top panel) and the polyrhythmic task (bottom panel). Model parameters were estimated simultaneously for both tasks for a tempo range of $1,400-8,200 \mathrm{~ms} / \mathrm{cycle}$ for each participant. The curve fits are based on averaged values for individual model estimates. $c_{00}=$ variance of the asynchrony $\mathrm{L}_{0} ; c_{11}=$ variance of interval $I_{1} ; c_{22}=$ variance of interval $I_{2} ;$ and so on.

source of variances for intervals $I_{1}$ to $I_{6}$, we would expect identical values for variances and covariances in this task (see Tables 1 and 2), leaving motor delay variances aside for the moment. A comparison of Figures 5 and 6 (top panels) reveals that this is clearly not the case. From the perspective of the rhythm program hypothesis, the absence of effects of timekeeper variability in the presence of marked effects of rate fluctuation implies that timekeepers execute variable target intervals with perfect accuracy, an unlikely scenario.
To directly compare the effects of timekeeper execution between tasks, we considered the two intervals with identical target durations in either task, namely intervals $I_{3}$ and $\mathbf{I}_{4}$ (see Figure 1). We conducted a repeated measures ANOVA using task (syncopated vs. polyrhythm), interval ( $\mathbf{I}_{3}$ vs. $\mathbf{L}_{4}$ ), and tempo (10 durations from $1,400 \mathrm{~ms} /$ cycle to 7,200 $\mathrm{ms} /$ cycle) as within-subjects factors. The only significant effects were related to the linear, $F(1,13)=147,55, M S E=$ $340,770, p<.001$, and quadratic, $F(1,13)=50.78, M S E=$ 
$120,195, p<.001$, increases in variances with cycle duration.

From these results it appears justified to fit the same model to the data from both tasks. The model equation for the variances are listed in Table 2 . We used the estimates for motor delay variances and rate fluctuation obtained earlier and estimated the three $p$ parameters by fitting the model to the variances of $I_{1}$ to $I_{6}$ observed in both tasks. Six variances were obtained at 10 different tempos $(1,400-7,200 \mathrm{~ms} /$ cycle) in each task, providing 120 observations for the estimation of three model parameters per participant. The curve fits in Figure 6 are based on estimates averaged across participants.

Evaluation of the integrated model for performance at slower tempos. Our analyses of performance at slower tempos provide ample support for the distinction between timekeeper and motor delay processes central to the extended two-level timing framework. The framework properly predicts the covariances that include the asynchrony in both tasks across a wide range of tempos. Wing (1980) estimated right-hand motor delay variances of $30 \mathrm{~ms}^{2}$ from a repetitive unimanual tapping task with isochronous intervals. He found this estimate to be largely invariant across target durations from 220 to $490 \mathrm{~ms}$. This value corresponds well to the integrated model's estimates $\left(31.09 \mathrm{~ms}^{2}\right)$ that we obtained for a much larger range of overall performance tempos. The increase in motor delay variances we observed in most participants probably has a straightforward explanation: Observation during the experiments and postexperimental interviews suggested that different types of movements, such as slow body swaying, came into play at slow tempos. Neither of these two findings warrant rejection of the extended two-level timing framework as far as the asynchrony and its covariances are concerned.

The extended two-level timing framework failed to account for the pattern of positive covariances between intervals other than the asynchrony unless additional processes were taken into account. Despite its relatively simple timekeeper structure (strict concatenation), the integrated timing model we proposed provides a reasonable approximation to the data from two rhythmic tasks performed over a wide range of tempos. $R^{2} \mathrm{~s}$ for model fits at the level of individual participants were acceptable, with a median $R^{2}$ of .85 ; 1 participant had an $R^{2}$ of .72 , and the values for the remaining pianists ranged between .80 and .90 .

The integrated model's account of the data has several limitations, some of which are obvious from Figure 6 . The variances of the longest intervals $\left(c_{11}\right.$ and $c_{66}$ ) in the polyrhythmic task were systematically underestimated; closer inspection reveals a (relatively smaller) overestimate for variances from the syncopated rhythm task, which becomes visible at medium tempo ranges in Figure 6. More detailed analyses revealed overall differences between the two tasks at faster $(1,400-4,800 \mathrm{~ms} / \mathrm{cycle})$ tempo conditions. The fit was substantially improved when we allowed $p$ and $q$ parameters in the model fits to differ between the two tasks (the identical timekeeper structure, strict concatenation, was maintained, however): Individual $R^{2} \mathrm{~s}$ ranged from .83 to .92 $(M d n=.87)$ in the syncopated rhythm task and from .83 to
$.93(M d n=.90)$ in the polyrhythmic task. Parameter values estimated suggest that especially timekeeper execution variability was higher at medium tempos in the polyrhythmic than in the control task.

One interpretation of this finding is that additional execution problems arise from a concatenated series of timekeepers with different target intervals. This assumption is plausible in light of earlier findings (Vorberg \& Hambuch, 1984). Related effects are probably minor compared with the dominant effects of rate fluctuations at the slowest tempos. None of our numerous modeling attempts supported different hierarchical timekeeper organizations for any of the two tasks. Related models predict systematic deviations of specific covariances from the overall pattern we found that were just not evident from our data. As an example, the variance of the last interval, $I_{6}$ that we observed in the polyrhythmic task was far below the values predicted from a hierarchical model with a superordinate timekeeper that controls the total length of the cycle. On these grounds, we decided to maintain identical models and identical parameters for both tasks for reasons of parsimony in our description.

A second systematic deviation between model predictions and data relates to the differences between adjacent and nonadjacent intervals. For adjacent interval pairs, our model predicts that positive covariation attributable to rate fluctuation must be reduced by an amount corresponding to the variance of the motor delay that separates the two intervals (see Table 1). At tempos of 1,400 to $4,200 \mathrm{~ms} / \mathrm{cycle}$, this prediction was almost perfectly met. At even slower rates, however, a systematic pattern of deviations from the model predictions emerged. Adjacent intervals and those separated only by one intervening interval tended to covary slightly higher than those separated by two, three, or more intervals within the rhythmic group. This phenomenon was similar in both tasks and suggests that our assumption of a constant rate within cycles is an approximation only at slow tempos. Instead, it is more likely that longer intervals are controlled by concatenated timekeeper signals as such and that local fluctuations in rate become more salient.

As a final point, the bottom panel in Figure 6 shows systematic differences in the polyrhythmic task between the variances of the second interval $\left(c_{11}\right)$ and the variance of the last interval $\left(c_{66}\right)$ in the cycle. Our model accounts for such findings on the basis of the mean realized intervals, which were in fact longer for $\mathrm{I}_{6}$ at tempos of $4,200 \mathrm{~ms} /$ cycle and above; however, the predicted differential effect was much smaller than we observed. One potential explanation is that participants prepared the simultaneous keystrokes during the final interval. However, we did not obtain similar differences in the other task, in which the requirements on finger sequencing were the same. Our guess is that pianists make adjustments on their overall performance rates during the last long interval in the polyrhythmic task.

At the bottom line, our findings for slow tempos support the predominant claim in the literature as far as integrated timing of polyrhythmic performance is concerned. At the start of our discussion of results, we presented qualitative evidence that suggests that different timing control struc- 
tures might be operating at slower and faster tempos. We now return to a closer look at the data from rapid performance rates.

\section{Timing at Rapid Tempos: The Parallel Model}

In this section we evaluate a simplified version of the parallel model for our data from rapid performance tempos. By simplified version, we mean that, at this point, we do not commit ourselves to a quantitative specification of the error correction mechanism but instead focus on the qualitative pattern of predictions that follow from the assumptions we outlined in the theory section. We consider the same intervals as in our discussion of timing at slower tempos, starting with the covariances between nonoverlapping intervals $I_{1}$ to $I_{6}$. We show that the checkerboard pattern of positive and negative covariation predicted by the parallel model does in fact emerge at rapid polyrhythmic performance. In this context we show that rate fluctuation can be ruled out as an account for the typical covariance pattern. We then demonstrate that timekeeper variance plays a negligible role at rapid rates and that the variance of the asynchrony is the major factor in explaining the variances of betweenhands intervals. Finally, we switch perspectives to the within-hands intervals for a direct demonstration of the differential benefits of integrated and parallel timing at fast and slow tempos.

Covariances among $I_{1}$ to $I_{6}:$ Checkerboard pattern caused by the asynchrony variance. To illustrate the potential effects of rate fluctuation on the covariances among $I_{1}$ to $I_{6}$, we consider the estimates for $\sigma_{X}^{2}$ for a cycle duration of 1,400 $\mathrm{ms}$, the fastest tempo evaluated on the "slow side." The model estimate is $266 \mathrm{~ms}^{2}$. From this value, the largest positive covariance introduced by rate fluctuation in the polyrhythmic task assuming ideal proportions (i.e., $c_{16}=$ $1 / 16 \sigma_{X}^{2}$ in Table 4) is in the range of $16-17 \mathrm{~ms}^{2}$. Even smaller values result if rate fluctuation continues to decrease at more rapid rates. This picture changes completely if the rate becomes more variable again at faster tempos. Positive covariation attributable to rate fluctuation must eventually cancel out negative covariation induced by shared motor delays between adjacent intervals, as we demonstrated for slower tempos.

We use the covariances including $I_{2}$ shown in Figure 7 for a more detailed illustration of these relations. The predicted effects of $\sigma_{0}^{2}$ are negative for $c_{23}$, positive for $c_{24}$, and negative for $\mathrm{c}_{25}$, and this was in fact the pattern that emerged for rapid polyrhythmic performance. Consistent with the predictions, covariances between adjacent intervals $\left(c_{23}\right.$ in Figure 7) were even more negative, which was due to the shared motor delay. Finally, $c_{26}$ did not depend on $\sigma_{0}^{2}$ according to the parallel model and is therefore diagnostic for the effects of rate fluctuation. This covariance assumes close to zero values rather than increasing with tempo.

Although a similar pattern emerged at the most rapid tempo in the syncopated rhythm task with its isochronous structure, the overall evidence for parallel timing in this task was clearly less compelling. A similar picture emerged when we considered the covariances related to the asynchrony for
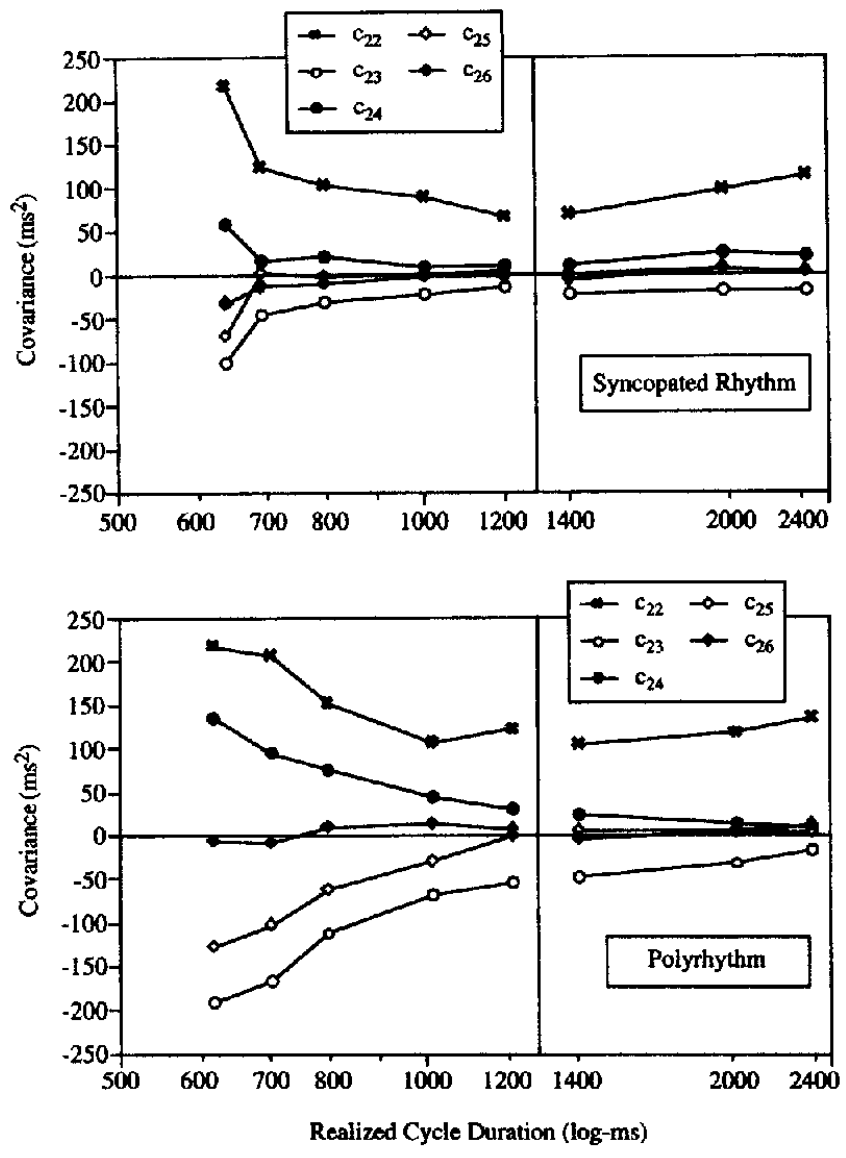

Figure 7. Covariances including Interval 2 as a function of realized cycle durations for the syncopated rhythm task (top panel) and the polyrhythmic task (bottom panel). The values in the left part of each panel are averaged from the 6 participants showing the best performances at rapid tempos. All 14 participants contributed to the data points at slower tempos depicted in the right sections of each panel. $c_{22}=$ variance of interval $I_{2} ; c_{23}$ to $c_{26}=$ covariance of interval $I_{2}$ with the following intervals in the cycle.

both tasks in Figure 3. The mean observed durations for all intervals and their covariances at rapid rates are provided in Table 6 for the syncopated rhythm task and in Table 7 for the polyrhythm. In the following section we focus on the polyrhythmic task. We then return to the results from the control task but refrain from a final evaluation of the evidence in this task until the end of the Results section.

Table 7 shows that the findings related to $I_{2}$ generalize to the complete covariance matrix in the polyrhythmic task. The most critical evidence for both the Wing-Kristofferson model and timekeeper models assuming integrated organization is the checkerboard pattern (i.e., the systematic occurrence of negative covariances at higher lags accompanied by higher order positive covariances in positions predicted by the parallel model). As the last column in Table 7 shows, this pattern did not extend to covariances with the last interval in the cycle, $I_{6}$, confirming another strong prediction of the parallel model. The fact that the predicted pattern was also evident for covariances related to the asynchrony directly 
Table 6

Mean Observed Interval Durations and Covariances at Rapid Rates in the Syncopated Task

\begin{tabular}{lrrrrrrr}
\hline $\begin{array}{c}\text { Metronome } \\
\text { cycle duration }\end{array}$ & $I_{0}$ & $I_{1}$ & $I_{2}$ & \multicolumn{1}{c}{$I_{3}$} & \multicolumn{1}{c}{$I_{4}$} & \multicolumn{1}{c}{$I_{5}$} & \multicolumn{1}{c}{$I_{6}$} \\
\hline 600 -ms duration & 3 & 113 & 98 & 104 & 109 & 82 & 130 \\
$\mathbf{I}_{0}$ & 223 & -170 & 104 & -52 & 19 & -35 & -60 \\
$I_{1}$ & & 220 & -159 & 70 & -18 & 20 & 57 \\
$I_{2}$ & & & 217 & -101 & 58 & -70 & -32 \\
$I_{3}$ & & & & 330 & -229 & 89 & -51 \\
$I_{4}$ & & & & & 266 & -153 & 72 \\
$I_{5}$ & & & & & & 288 & -103 \\
$I_{6}$ & 3 & 119 & 113 & 114 & 114 & 98 & 131 \\
$700-m s$ duration & 137 & -104 & 46 & -15 & -6 & 6 & -13 \\
$I_{0}$ & & 159 & -97 & 19 & -4 & -10 & 11 \\
$I_{1}$ & & & 123 & -45 & 17 & 2 & -13 \\
$I_{2}$ & & & & 154 & -88 & 11 & -4 \\
$I_{3}$ & & & & & 120 & -56 & 12 \\
$I_{4}$ & & & & & & 141 & -60 \\
$I_{5}$ & 0 & 137 & 131 & 133 & 134 & 117 & 148 \\
$I_{6}$ & 100 & -76 & 35 & -10 & 7 & -8 & -6 \\
800 -ms duration & 126 & -75 & 12 & -11 & 1 & 9 \\
$I_{0}$ & & & 102 & -31 & 21 & -2 & -10 \\
$I_{1}$ & & & & 88 & -47 & 15 & -3 \\
$I_{2}$ & & & & & 71 & -33 & 8 \\
$I_{3}$ & & & & & & 89 & -37 \\
$I_{4}$ & & & & & & & 80 \\
$I_{5}$ & & & & & & \\
$I_{6}$ & & & & & & & \\
\hline
\end{tabular}

Note. I = interval.

rules out a complete class of integrated timing models with hierarchical timekeeper structures as an account of our data.

More detailed consideration of covariances between adjacent intervals (first off-diagonal in Table 7) illustrates further evidence for the parallel model. Consistent with the assumptions of two-level timing, the covariances between adjacent intervals were negative. However, all covariances among adjacent pairs of intervals $I_{0}$ to $I_{5}$ correlated negatively with values exceeding the -.5 boundary imposed by the extended two-level timing framework: The corresponding values ranged from -.570 to -.903 for those durations shown in Table 7. This finding is clear evidence for influences over and above the motor delay level as implied by the parallel model. In contrast, standardized values of $c_{56}$ $(-.06$ to -.26$)$ were still negative but were much smaller in their absolute sizes. The parallel model's predictions for $c_{56}$ were basically identical to those from the two-level timing framework in that $c_{56}$ reflected motor delay variances. In line with the evidence reported earlier, the negative values for $c_{56}$ suggest that residual influences of rate fluctuation cannot cancel out negative covariation attributable to motor delays at rapid tempos. We found the same systematic differences among adjacent intervals for the two tempos (1,000 and $1,200 \mathrm{~ms} / \mathrm{cycle}$ ) not listed in the tables for reasons of space limitations. Related correlations were negative but attenuated. We attribute this finding to the increasing influences of rate fluctuation with longer target durations.

As a further evaluation of the parallel model's explanatory scope, we calculated the standardized covariances (see Footnote 3 ) among intervals $I_{0}$ to $I_{5}$ for each individual participant; we then converted these values into discrete symbols reflecting positive or negative correlations, respectively. Figure 8 shows that the chessboard pattern predicted from the parallel model did in fact emerge at the level of individual participants. We have extended this illustration by including our best 5 participants' performances at the 500-ms/cycle tempo. If we apply sign-test criteria instead of the cutoff values of -.1 and +.1 used in Figure 8, we obtain perfect patterns for tempos of $500-800 \mathrm{~ms} / \mathrm{cycle}$ for all 6 participants; the number of violations at a cycle duration of $1,000 \mathrm{~ms}$ was effectively cut by half.

Variances of $I_{1}$ to $I_{6}$. In extending our considerations of the variances of $I_{1}$ to $I_{6}$, we assume that the effects of timekeeper execution were also minimal for the rapid rates considered here. Under these assumptions, the parallel model predicted that the variances of between-hands intervals $I_{1}$ to $I_{5}$ would depend on $\sigma_{0}^{2}$ for the most part and show the same changes with tempo as $\sigma_{0}^{2}$. The notable exception should be $\mathrm{c}_{66}$, the variance of $\mathrm{I}_{6}$, which was not affected by the asynchrony.

Figure 9 illustrates that the data in the polyrhythmic task matched the predictions of the parallel model: Although the variance of the asynchrony (denoted as $c_{00}$ in Figure 9) and $c_{11}$ to $c_{55}$ increased correspondingly, $c_{66}$ continued to decrease with tempo. Consistent with the evidence obtained from the covariances between intervals, this finding reflects a further decrease of rate fluctuation at even more rapid rates, and the same argument can be applied to the variability during timekeeper execution. Note that the pattern of variances obtained for rapid polyrhythmic performance is an

Table 7

Mean Observed Interval Durations and Covariances at Rapid Rates in the Polyrhythmic Task

\begin{tabular}{|c|c|c|c|c|c|c|c|}
\hline $\begin{array}{l}\text { Metronome } \\
\text { cycle duration }\end{array}$ & $\mathbf{I}_{0}$ & $I_{1}$ & $I_{2}$ & $I_{3}$ & $\mathrm{I}_{4}$ & $\mathrm{I}_{5}$ & $I_{6}$ \\
\hline $\begin{array}{c}\text { 600-ms duration } \\
I_{0} \\
I_{1} \\
I_{2} \\
I_{3} \\
I_{4} \\
I_{5} \\
I_{6}\end{array}$ & $\begin{array}{r}1 \\
194\end{array}$ & $\begin{array}{r}152 \\
-177 \\
198\end{array}$ & $\begin{array}{r}62 \\
132 \\
-144 \\
218\end{array}$ & $\begin{array}{r}92 \\
-121 \\
132 \\
-191 \\
217\end{array}$ & $\begin{array}{r}109 \\
89 \\
-90 \\
135 \\
-148 \\
204\end{array}$ & $\begin{array}{r}49 \\
-83 \\
84 \\
-128 \\
135 \\
-197 \\
231\end{array}$ & $\begin{array}{r}154 \\
-4 \\
-1 \\
-6 \\
-4 \\
3 \\
-6 \\
45\end{array}$ \\
\hline $\begin{array}{c}700 \text {-ms duration } \\
I_{0} \\
I_{1} \\
I_{2} \\
I_{3} \\
I_{4} \\
I_{5} \\
I_{6}\end{array}$ & $\begin{array}{l}-8 \\
147\end{array}$ & $\begin{array}{r}178 \\
-136 \\
162\end{array}$ & $\begin{array}{r}67 \\
103 \\
-128 \\
207\end{array}$ & $\begin{array}{r}109 \\
-86 \\
104 \\
-167 \\
177\end{array}$ & $\begin{array}{r}119 \\
39 \\
-50 \\
95 \\
-103 \\
150\end{array}$ & $\begin{array}{r}63 \\
-44 \\
57 \\
-102 \\
105 \\
-140 \\
170\end{array}$ & $\begin{array}{r}176 \\
-11 \\
11 \\
-8 \\
9 \\
10 \\
-16 \\
47\end{array}$ \\
\hline $\begin{array}{l}800 \text {-ms duration } \\
\mathrm{I}_{0} \\
\mathrm{I}_{1} \\
\mathrm{I}_{2} \\
\mathrm{I}_{3} \\
\mathrm{I}_{4} \\
\mathrm{I}_{5} \\
\mathrm{I}_{6}\end{array}$ & $\begin{array}{l}-9 \\
100\end{array}$ & $\begin{array}{r}203 \\
-78 \\
108\end{array}$ & $\begin{array}{r}77 \\
50 \\
-73 \\
152\end{array}$ & $\begin{array}{r}124 \\
\mathrm{y} 37 \\
55 \\
-112 \\
123\end{array}$ & $\begin{array}{r}134 \\
20 \\
-29 \\
76 \\
-80 \\
116\end{array}$ & $\begin{array}{r}75 \\
-15 \\
28 \\
-62 \\
63 \\
-98 \\
139\end{array}$ & $\begin{array}{r}196 \\
0 \\
0 \\
10 \\
-7 \\
18 \\
-22 \\
51\end{array}$ \\
\hline
\end{tabular}




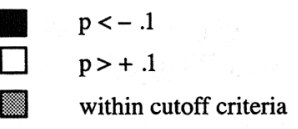

Metronome Cycle Duration $=500 \mathrm{~ms} ; 0$ violations out of 75
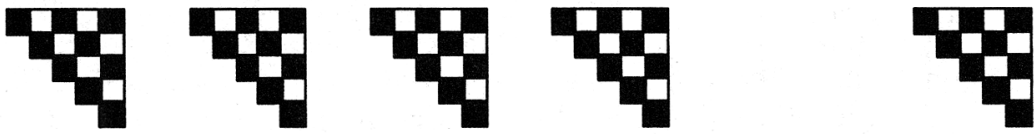

Metronome Cycle Duration $=600 \mathrm{~ms} ; 1$ violation out of 90
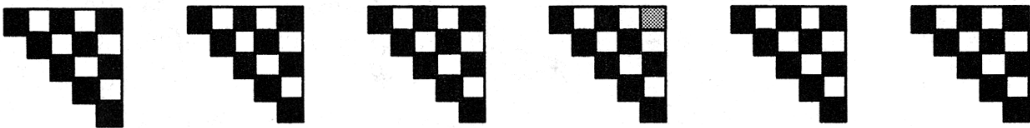

Metronome Cycle Duration $=700 \mathrm{~ms} ; 0$ violations out of 90
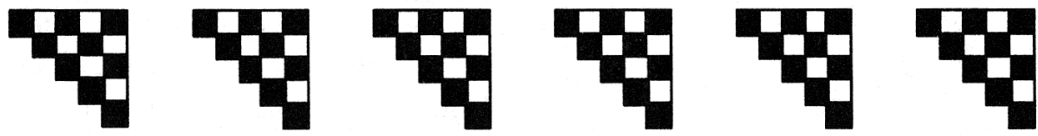

Metronome Cycle Duration $=800 \mathrm{~ms} ; 9$ violations out of 90
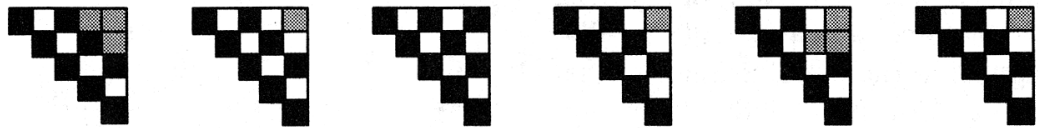

Metronome Cycle Duration $=1000$ ms; 20 violations out of 90
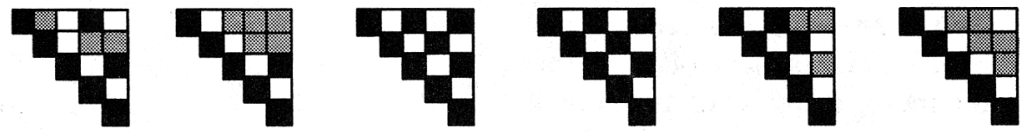

Figure 8. Checkerboard pattern of negative (black) and positive (white) correlations (corr) between Intervals $0-5$ for the 6 best participants' polyrhythmic performance. Values falling within the cutoff criteria are marked as gray squares and are included in our count of violations from model predictions. The order of symbols is $I_{0}$ to $I_{5}$ (i.e., the top line in each board refers to corr 01 to corr 05 ; Line 2 denotes $\operatorname{corr}_{12}$ to $\operatorname{corr}_{15}$; and the bottom symbol in each board is $\operatorname{cor}_{45}$ ).

exception to the usual dependence between means and variances; as predicted by the model, the size of the variances was dominated by the asynchrony variance rather than reflecting differences in mean durations. At slower tempos, the variances in the polyrhythmic task (right part of the bottom panel in Figure 9) separated out, reflecting the different target values of long $\left(c_{11}\right.$ and $\left.c_{66}\right)$, median $\left(c_{33}\right.$ and $\left.c_{44}\right)$, and short $\left(c_{22}\right.$ and $\left.c_{55}\right)$ intervals.

The data from the syncopated rhythm task revealed a different pattern: First, $c_{66}$ was smaller than the other covariances but showed the same increase with tempo. Second, the variances fanned out at the most rapid tempo, with $c_{33}$ to $c_{55}$ having larger values than the remaining variances of intervals from the same task and the corresponding intervals with similar means in the polyrhythmic task. One explanation for the overall increase in variances is that performance in the syncopation task remained subject to integrated timing at all tempos. 

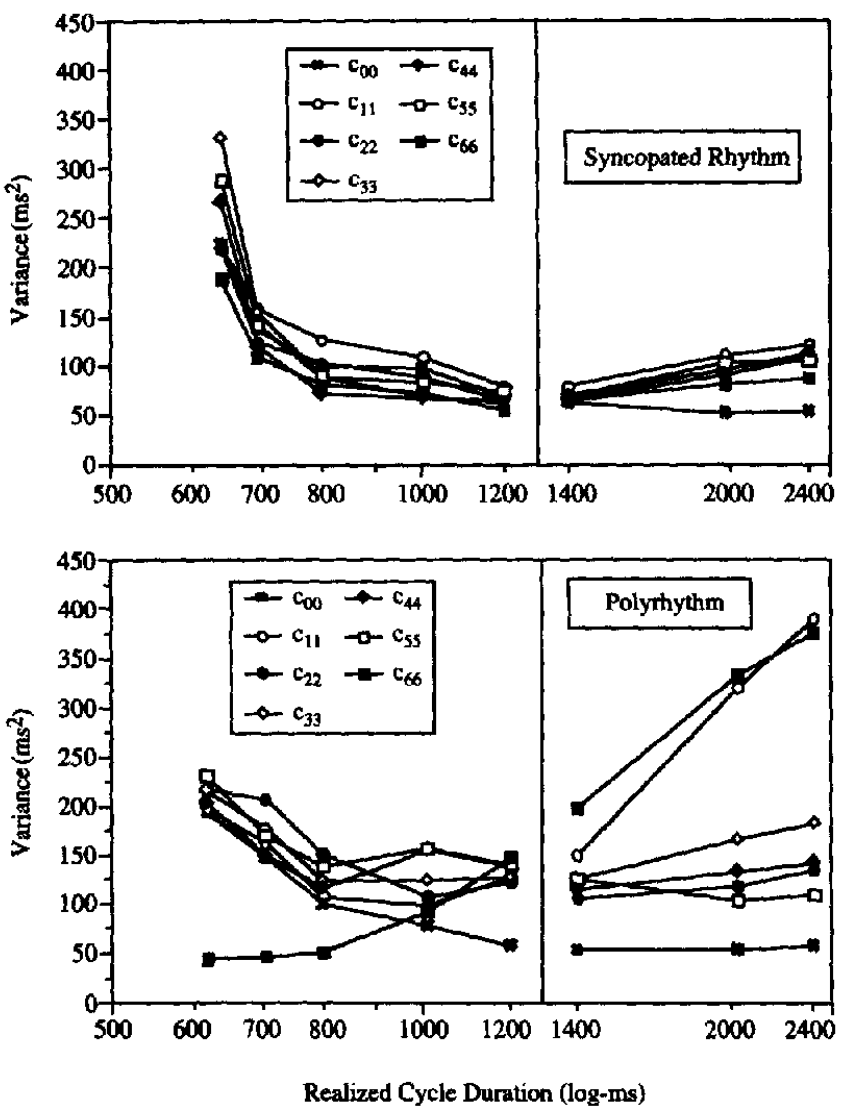

Figure 9. Variances of between-hands Intervals $0-6$ as a function of realized cycle durations for the syncopated rhythm task (top panel) and the polyrhythmic task (bottom panel). The values in the left part of each panel are averaged from the 6 participants showing the best performances at rapid tempos. All 14 participants contributed to the data points at slower tempos depicted in the right sections of each panel. $c_{00}=$ variance of the asynchrony $I_{0} ; c_{11}=$ variance of interval $I_{1} ; c_{22}=$ variance of interval $I_{2}$; and so on.

There is, however, one important difference between the two tasks that suggests an alternative explanation. In the syncopated rhythm, left within-hands intervals are isochronous but right within-hands intervals are not. We argued before that parallel timing is a means by which experts can acclimate to the performance constraints arising from rapid rates and that the polyrhythmic task is more suited for parallel timing because of the isochronous structure for within-hands intervals in both hands. Likewise, integrated timing should benefit from the isochronous structure of between-hands intervals that is characteristic of the syncopated rhythm task. It is this very aspect we aimed at in our most far-reaching prediction: At slower tempos, accuracy should be higher (i.e., variability of observed intervals should be lower) in the syncopated thythm tasks than in the polyrhythm tasks because of integrated timing; in contrast, polyrhythmic performance at rapid tempos is actually easier (i.e., it shows higher accuracy) than for the syncopation task, with its much simpler rhythmic structure. In the final part of the
Results section, we switch perspectives to within-hands intervals to evaluate this claim.

\section{Costs and Benefits of Integrated and Parallel Timing: Variances of Within-Hands Intervals}

A different description of the adaptive character of parallel timing is that pianists accept more variability in the starting and end points of parallel streams (i.e., the asynchrony) to permit greater hand independence within rhythmic cycles. There is one critical implication to this perspective: Although variances of between-hands intervals must increase to the degree that the variance of the asynchrony increases, the variance of within-hands intervals can be kept small and, ideally, can continue to decrease with tempo even at extreme rates. Figures 10 and 11 show the variances
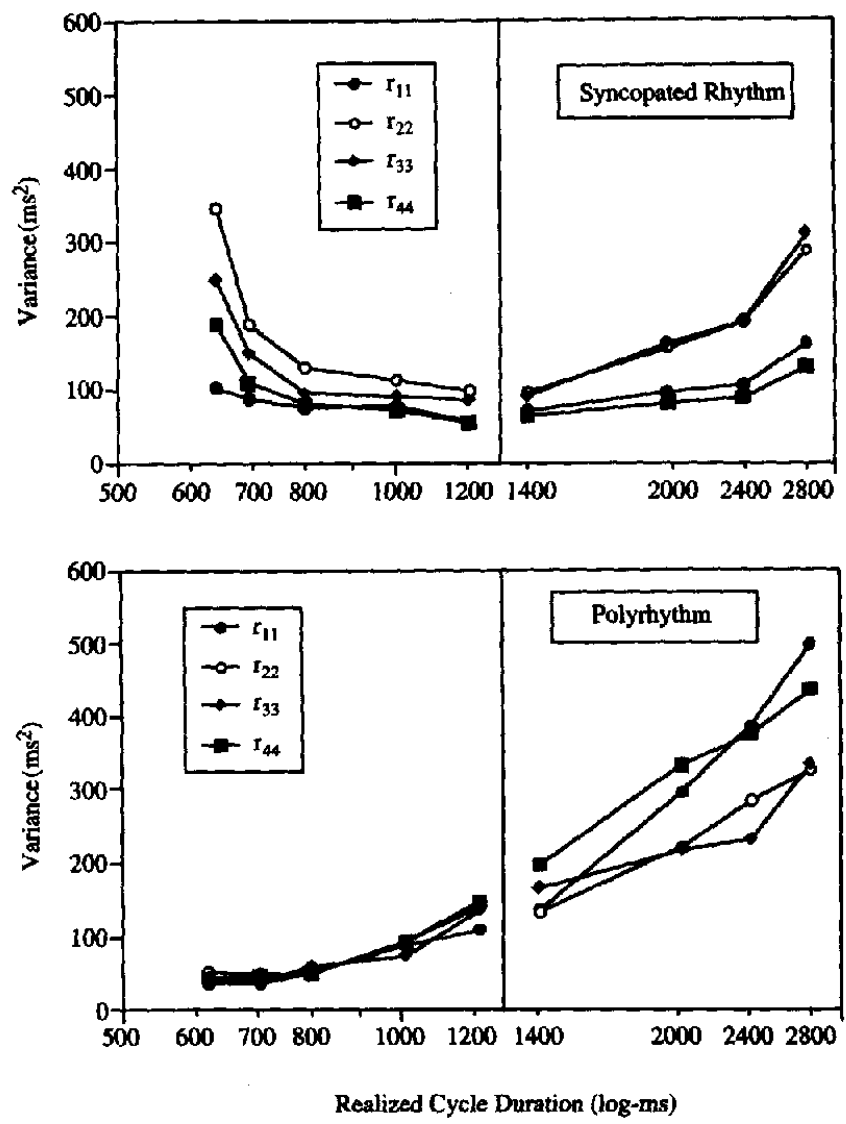

Figure 10. Variances of right within-hands intervals as a function of realized cycle durations for the syncopated rhythm task (top panel) and the polyrhythmic task (bottom panel). Right withinhands intervals form an anisochronous sequence in the syncopated rhythm with $R_{1}$ and $R_{4}$ having half the ideal target duration of $R_{2}$ and $\mathbf{R}_{\mathbf{3}}$. In the polyrhythmic task, right within-hands intervals form an isochronous sequence. The values in the left part of each panel are averaged from the 6 participants showing the best performances at rapid tempos. All 14 participants contributed to the data points at slower tempos depicted in the right sections of each panel. $r_{11}=$ the variance of the first right hand interval $R_{1} ; r_{22}=$ the variance of the second right hand interval $\mathbf{R}_{2}$; and so on. 

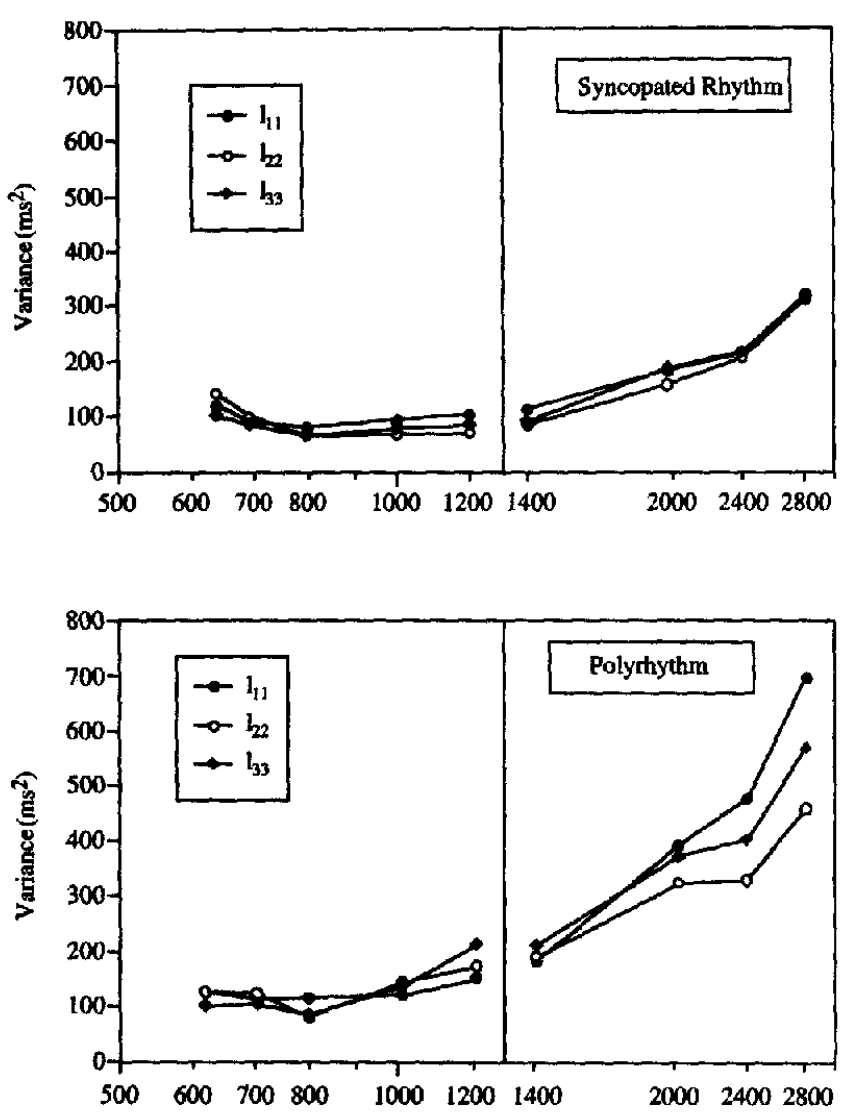

Realized Cycle Duration (log-ms)

Figure 11. Variances of left within-hands intervals as a function of realized cycle durations for the syncopated rhythm task (top panel) and the polyrhythmic task (bottom panel). Ideal durations for left within-hands intervals are identical in both tasks and form isochronous sequences. The values in the left part of each panel are averaged from the 6 participants showing the best performances at rapid tempos. All 14 participants contributed to the data points at slower tempos depicted in the right sections of each panel. $1_{11}=$ the variance of the first left hand interval $L_{1} ; l_{22}=$ the variance of the second left hand interval $L_{2} ; l_{33}$ is the variance of the last left hand interval $\mathrm{L}_{3}$.

calculated for right and left within-hands intervals, respectively. ${ }^{4}$ Consistent with our argument, variances in both hands decrease or level off at higher tempos in the polyrhythmic task. Direct support for the claim that the polyrhythmic task is in fact easier at extreme tempos comes from a comparison of right within-hands variances across tasks. In the syncopation task, these variances (left part of the top panel in Figure 10) increase with tempo. Values for tempos of $800 \mathrm{~ms} / \mathrm{cycle}$ and faster are well above those in the polyrhythmic task regardless of which interval is considered.

A comparison of rapid and slower tempos in Figures 10 and 11 illustrates the adaptive value of using different timing control modes for different tempos. Overall, the right within-hands variances in the syncopation task reflect the mean realized target durations in a similar manner for fast and slow tempos; $r_{22}$ and $r_{33}$ refer to intervals with twice the ideal target value compared with $r_{11}$ and $r_{44}$ (see Figure 2). Table 6 shows that participants systematically deviated from ideal values at $600 \mathrm{~ms} / \mathrm{cycle}$, presumably trying to partly compensate for the biomechanical constraints imposed by the right-hand repetitions delimiting $R_{1}$ and $R_{4}$.

In slow polyrhythmic performance (right part of bottom panel in Figure 10) $r_{11}$ and $r_{44}$ differed systematically from $r_{22}$ and $r_{33}$, although the mean realized durations for the four within-hands intervals were almost identical. This is what the integrated model predicts because $R_{1}$ and $R_{4}$ are controlled by single timekeepers, whereas $R_{2}$ and $R_{3}$ emerge from concatenation, which in turn reduces the variability of the within-hands intervals. In contrast, the four covariances lie on top of each other for rapid polyrhythmic performance, a finding that we attributed to parallel timing.

The situation is more complicated for the left withinhands intervals. The smallest variance on the slow side of the performance function (right part of bottom panel in Figure 11) refers to interval $\mathrm{L}_{2}$. Figure 2 shows that in the integrated model, this interval emerges from the concatenation of two timekeepers (C and D) that delineate identical (i.e., isochronous) target intervals. As we argued before, this is easier than for the concatenation of timekeepers with different target intervals, as is the case for $L_{1}$ and $L_{3}$ in our model. This interpretation was confirmed by the finding that the corresponding variances $1_{11}, 1_{22}$, and $\mathrm{l}_{33}$ did not differ from each other to the same degree in the syncopated rhythm task.

A comparison of variances for right and left within-hands intervals in the syncopated rhythm task shows that the right hand suffered overproportionally from increases in tempo. This is to be expected from the anisochronous sequence to be performed by the right hand in this task if parallel timing is attempted. In postexperimental interviews, 2 of our 6 best experts pointed out that the only way they could perform the syncopated rhythm task at tempos such as $600 \mathrm{~ms} / \mathrm{cycle}$ was to play the described right-hand pattern as an independent figure in parallel to the left hand, which they deemed much more complicated than the polyrhythm. Presumably, the rapid tempo data in the syncopated rhythm task represent a mixture of timing control structures, including participants' unsuccessful attempts to switch to a parallel mode or a lack of stability in their timing control.

All 14 participants claimed to perform the polyrhythm in a hand-independent manner at tempos of $800 \mathrm{~ms} / \mathrm{cycle}$ or faster, and our data demonstrate that 6 pianists in fact did so for tempos of up to $600 \mathrm{~ms} / \mathrm{cycle}$. Because of successful implementation of parallel timing, these experts eventually

\footnotetext{
${ }^{4}$ Mean realized durations for single within-hands intervals can be added up from successive nonoverlapping intervals $I_{0}$ to $I_{6}$, which we report for rapid tempos in Tables 5 and 6 . Because of the distributivity of the covariance, within-hands variances can be computed as sums of covariances between $I_{0}$ and $I_{6}$. There is one important exception, however: Calculation of $L_{33}$, the variance of the last left-hand interval, involves covariances $c \xi_{0}$ and $c *_{0}$ because it includes the asynchrony at cycle-lag 1 .
} 
attained a higher accuracy in the polyrhythmic task than in the control task with its simple isochronous structure. Besides participants' self-reports, other empirical findings support our interpretation of reversed task difficulty effects at rapid tempos: (a) None of our participants could perform the syncopated rhythm task in the $500-\mathrm{ms} /$ cycle condition, whereas 5 participants could do so in the polyrhythmic task; (b) the number of acceptable trials provided by our best participants at a tempo of $600 \mathrm{~ms} / \mathrm{cycle}$ was higher in the polyrhythmic than control task; and (c) during these trials the mean realized tempo was $21 \mathrm{~ms}$ closer to the ideal (i.e., faster) in the polyrhythmic task.

\section{Discussion}

Consistent with earlier studies (Deutsch, 1983; Jagacinski et al., 1988; Klapp, 1979; Summers \& Pressing, 1994), we found that pianists relied on integrated timing control at slower tempos. This was equally true for the polyrhythmic task, the structure of which is most suited to encourage parallel timing. At fast tempos, we found that the pianists used parallel timing for the hands, thereby permitting partial hand independence in polyrhythmic performance. Our evidence is incompatible with the assumption that a single programmable clock times successive nonoverlapping intervals and triggers both hands simultaneously or in alternation.

Our findings provide ample support for the two-level timing distinction originally proposed by Wing and Kristofferson (1973a). At the same time, we showed that the concept of a single programmable clock cannot account for the covariance structure in rhythmic timing tasks, and this was true for the complete range of tempos investigated. In that respect, our data replicate and extend earlier findings by Vorberg and Hambuch $(1978,1984)$. We proposed a timermotor model for performance at slower tempos that we developed in the context of the rhythm program hypothesis (Vorberg \& Wing, 1996). Our model gives a quantitative account for the data from two diverse rhythmic tasks performed over a wide range of tempos. The concatenated timekeeper architecture underlying our model is in fact relatively simple, and the structure is not hierarchical in the strict sense. Although this may be less surprising in the case of the syncopated rhythm task with its isochronous structure, we showed that the same model provides a reasonable account of performance in the polyrhythmic task.

Our study provides what we believe is the first empirical test of the central theoretical concept inherent to the rhythm program framework, namely the distinction between target specification and timekeeper execution. In this context we were able to resolve a puzzle that emerged from earlier studies: the absence of the negative lag-1 autocorrelation at slower tempos that is especially apparent in musical performance (Repp, 1999). Our data show that this finding may not be attributed to a lack of biomechanical constraints at slow tempos but instead reflects the increasing role of rate fluctuation during the production of longer intervals.

Besides its plausibility from a musical performance point of view (changing tempo while preserving the overall pattern), the distinction between target specification and timekeeper execution avoids the well-known problems of the invariant-relative timing notion originally proposed in the context of general motor programming (for discussions, see Gentner, 1987; Heuer, 1988, 1991). Related arguments for separate mechanisms for timing and serial order control have been made from a neuropsychological perspective. Keele, Cohen, and Ivry (1990) discussed a number of experimental and neurological studies that point to distinct modules for timing and sequence representation. The basic distinction of the two components is also central to MacKay's (1987) model of skilled performance.

Our parallel model has several distinct features from which we derived qualitative predictions. These predictions were met almost perfectly in the polyrhythmic task. The checkerboard pattern characteristic of the off-diagonal covariance matrix is highly discriminative with respect distinguishing parallel and integrated control. We were able to demonstrate the pattern at the level of individual participants. Vorberg and Wing (1996) showed theoretically that complex control structures with timekeepers operating at different levels in a hierarchy can produce positive and negative covariation in the same task even if integrated timing is assumed. Related models have been proposed by Jagacinski et al. (1988). However, all integrated models including hierarchical timekeeper structures must predict zero covariances with the asynchrony at higher lags; this prediction was violated in our data in a systematic manner, supporting our assumption of parallel timing. Furthermore, we showed that rate fluctuation plays a major role at slower tempos but decreases at more rapid tempos to a degree that it can be ruled out as an explanation for positive covariances.

At a quantitative level, the parallel model we proposed is as yet incomplete in that it lacks a detailed specification of the error correction or coupling process. We have proposed that error correction is restricted to initiating or terminating the next rhythmic cycle earlier or later, respectively, in response to the previous asynchrony. From our data, the assumption that, within rhythmic cycles, production of within-hands intervals proceeds in a parallel open-loop fashion provides a reasonable approximation. More precise formulations, however, require further empirical investigation and modeling. Certain patterns in our data provide hints in that respect, such as a tendency for covariances to decrease in magnitude with distance from the asynchrony. One explanation for this pattern could be that the hands gradually drift apart within rhythmic groups. A candidate model would be a parallel architecture that assumes different rates for the two hands. At this point, however, our model is not sufficiently developed to capture all details of the timing control mechanisms at rapid tempos. Whatever the precise nature of a timing model will be, it must incorporate the parallel architecture we demonstrated.

\section{Alternative Accounts}

Recently, Helmuth and Ivry (1996) provided evidence from synchronized bimanual performance that is also diffi- 
cult to reconcile with the assumption of a single central clock. They demonstrated a reduction of variability in the synchronized bimanual as compared with the unimanual case for both hands. It is important that this effect cannot be explained by an improvement of the weak (left) hand as such. Helmuth and Ivry explained the variance reduction in the bimanual tasks as being the result of an averaging process based on separate timekeepers for the two hands that yields a higher accuracy than the output of a single mechanism. Rather than assuming a single clock, like in Wing and Kristofferson's (1973a) model, Helmuth and Ivry proposed that the central timing device consists of multiple timekeepers. According to the authors, the constraints on bimanual performance reflect a response bottleneck; output to the different effector system does not occur continuously but is gated and emitted with limited frequency. Assuming that bimanual performance imposes extreme constraints on the gating mechanism, the system output can appear to be similar to one produced by a single clock under these circumstances. Following this approach, our data suggest that pianists' ability to maintain parallel timing reflects a higher temporal resolution of their gating mechanisms.

Different from earlier modeling work on polyrhythmic performance (Jagacinski et al., 1988; Pressing et al., 1996), we deliberately chose a continuation paradigm rather than having participants play in synchrony with a metronome. We consider the necessity to synchronize with an external metronome as a specific situation (for a discussion, see Vorberg \& Wing, 1996), the demands of which might prevent complete use of participants' timing and coordination capabilities. At the same time, synchronization with a metronome can effectively eliminate the effects of rate fluctuation. The findings presented by Pressing et al. point to a systematic subordination of one hand under the other. This referential timing is probably mandatory in a synchronization paradigm because participants would have a hard time simultaneously maintaining parallel streams and synchronizing each stream with the respective external pacing signals. In related models, the covariances related to intervals from the subordinated hands are limited to its adjacent intervals. Such a model cannot account for the higher order covariances we found. The figure-ground distinction proposed by Pressing et al. is a promising concept at a more general level. Our pianists reported that they focused more on one hand than on the other, leaving the less attended hand to "run on autopilot" during rapid performance. Interestingly, participants differed with respect to which hand they focused on. This kind of description is in fact perfectly compatible with the assumption of parallel timing and the figure-ground distinction. The effects of attention in bimanual tasks have also been demonstrated in earlier work by Peters (1981; Peters \& Schwartz, 1989).

The study of bimanual coordination has featured prominently in the context of the dynamic systems approach (Haken, Kelso, \& Bunz, 1985; Peper, Beek, \& van Wieringen, 1995; Yamanishi, Kawato, \& Suzuki, 1980). In this framework, multiple oscillators are genuine to the system, and bimanual coordination is attributed to the status of an emergent property in a dynamical system that is described completely in physical terms. Although this approach is highly elegant or parsimonious from a mathematical perspective, it is neutral or underspecified with respect to psychological mechanisms and their neural basis. The two-level timing concept is supported by a considerable number of experimental and neuropsychological studies, but it still has no specific meaning in a dynamic systems framework. A similar argument can be made for the distinction between serial order control and timekeeper execution. At this point, it is difficult to evaluate the correspondence between dynamic models and timer-motor models, partly because methods from alternative approaches are rarely considered together or contrasted with each other. More recently, there have been attempts to this end (e.g., Jordan \& Rosenbaum, 1989; Pressing, 1995; Turvey, Schmidt, \& Rosenblum, 1989). In the long run, oscillator concepts hold promise as models for specific modules in a more complex architecture. Intriguing examples for this perspective come from neurophysiological models of animal movements (Cattaert, Barthe, \& Clarac, 1994).

Manipulations of auditory feedback delay (Wing, 1977) and studies with deafferented patients (Ivry, Keele, \& Diener, 1988; Keele \& Ivry, 1991) have demonstrated that the human motor system can in principle function in an open-loop manner in simple timing tasks and that the classic closed-loop approach in its simplest form is wrong. In complex rhythm production, however, auditory feedback might play a different role, especially at rapid tempos. Our data illustrate that another tenet of the two-level timing framework must be given up eventually, namely the assumption that timing proceeds in a perfectly open-loop manner at all tempos. One possible extension of the parallel model relates to the role of feedback at the end of each cycle, when the two hands are combined. Any conceivable error correction presumes that participants use internal feedback, external feedback, or both to keep asynchronies small. Engbert et al. (1997) have proposed that feedback and error correction in thythm production might become effective with a certain time delay. This model avoids many problems of the classical open-loop approach.

The piano keys our participants used to produce the rhythms were one octave apart, and the tones were highly distinguishable. At faster tempos, perceptual grouping of the two isochronous sequences into separate streams might actually become easier. Several participants reported a kind of gestalt-switch phenomenon from an anisochronous sequence to a genuine, polyrhythmic pattern at faster tempos. These, admittedly informal, observations highlight the need for further investigation of perceptual-motor interactions, especially the role of feedback during the performance of more complex timing tasks (Jagacinski et al., 1988; Jones, Jagacinski, Yee, Floyd, \& Klapp, 1995; Klapp et al., 1985).

\section{Conclusions}

We have argued that hand independence constitutes a distinct case for the adaptivity of human movement control 
that emerges at high levels of skill. From our perspective that expertise amounts to circumventing the limitations typical of nonexpert processing, we have made a case for the high flexibility of skilled performance. None of the present frameworks and models is sufficiently developed to capture the whole scope of this flexibility, and our modeling approach is at best one step in that direction. We have pointed out several directions, such as the role of auditory feedback and its differential effects depending on task complexity and tempo that, until more recently, have received too little attention. Another issue is whether the shift between modes of timing control is a passive response to changing performance constraints or whether it can be deliberately controlled by the individual. Bridging the different approaches and incorporating this type of human flexibility into a theoretical model will be the major challenge for future work.

\section{References}

Cattaert, D., Barthe, J.-Y., \& Clarac, F. (1994). Sensory-motor coordination in crustacean limbs during locomotion. In $\mathbf{S}$. Swinnen, H. Heuer, J. Massion, \& P. Casaer (Eds.), Interlimb coordination: Neural, dynamic, and cognitive constraints (pp. 49-73). San Diego, CA: Academic Press.

Chase, W. G., \& Ericsson, K. A. (1982). Skill and working memory. Psychology of Learning and Motivation, 16, 1-58.

Collier, G. L., \& Wright, C. E. (1995). Temporal rescaling of simple and complex ratios in thythmic tapping. Journal of Experimental Psychology: Human Perception and Performance, 23, 602-627.

Deutsch, D. (1983). The generation of two isochronous sequences in parallel. Perception \& Psychophysics, 34, 331-337.

Engbert, R., Scheffczyk, C., Krampe, R. T., Rosenblum, M., Kurths, J., \& Kliegl, R. (1997). Tempo-induced transitions in polyrhythmic hand movements. Physical Review E, 56(5), 56-66.

Ericsson, K. A., \& Charness, N. (1994). Expert performance: Its structure and acquisition. American Psychologist, 49, 725-747.

Ericsson, K. A., Krampe, R. T., \& Tesch-Römer, C. (1993). The role of deliberate practice in the acquisition of expert performance. Psychological Review, 100, 363-406.

Gentner, D. G. (1987). Timing and skilled motor performance: Tests of the proportional duration model. Psychological Review, 94, 255-276.

Gentner, D. R. (1988). Expertise in typewriting. In M. T. H. Chi, R. Glaser, \& M. J. Farr (Eds.), The nature of expertise (pp. 1-21). Hillsdale, NJ: Erlbaum.

Haken, H., Kelso, J. A. S., \& Bunz, H. (1985). A theoretical model of phase transition in human hand movements. Biological Cybernetics, 51, 347-356.

Helmuth, L. L., \& Ivry, R. B. (1996). When two hands are better than one: Reduced timing variability during bimanual movement. Journal of Experimental Psychology: Human Perception and Performance, 22, 278-293.

Heuer, H. (1988). Testing the invariance of relative timing: Comment on Gentner (1987). Psychological Review, 95, 552-557.

Heuer, H. (1991). Invariant relative timing in motor-program theory. In J. Fagard \& P. H. Wolff (Eds.), The development of timing control and temporal organization in coordinated action (pp. 37-68). Amsterdam: North-Holland.

Hoaglin, D. C., Mosteller, F., \& Tukey, J. W. (1983). Understanding robust and exploratory data analysis. New York: Wiley.
Ivry, R. B., \& Hazeltine, R. E. (1995). Perception and production of temporal intervals across a range of durations: Evidence for a common timing mechanism. Joumal of Experimental Psychology: Human Perception and Performance, 21, 3-18.

Ivry, R. B., Keele, S. W., \& Diener, H. C. (1988). Dissociation of the lateral and medial cerebellum in movement timing and movement execution. Experimental Brain Reseanch, 73, 167-180.

Jagacinski, R. J., Marshburn, E., Klapp, S. T., \& Jones, M. R. (1988). Tests of parallel versus integrated structure in polyrhythmic tapping. Journal of Motor Behavior, 20, 416-442.

Jones, M. R., Jagacinski, R. J., Yee, W., Floyd, R. L., \& Klapp, S. T. (1995). Tests of attentional flexibility in listening to polyrhythmic patterns. Journal of Experimental Psychology: Human Penception and Performance, 21, 293-307.

Jordan, M. I., \& Rosenbaum, D. A. (1989). Action. In M. I. Posner (Ed.), Foundations of cognitive science (pp. 727-767). Cambridge, MA: MIT Press.

Keele, S. W., Cohen, A., \& Ivry, R. (1990). Motor programs: Concepts and issues. In M. Jeannerod (Ed.), Attention and performance XIII: Motor representation and control (pp. 77109). Hillsdale, NJ: Earlbaum.

Keele, S. W., \& Ivry, R. (1991). Does the cerebellum provide a common computation for diverse tasks? Annals of the New York Academy of Sciences, 608, 179-211.

Keele, S. W., Pokorny, R. A., Corcos, D. M., \& Ivry, R. (1985). Do perception and motor production share common timing mechanisms: A correlational analysis. Acta Psychologica, 60, 173-191.

Klapp, S. T. (1979). Doing two things at once: The role of temporal compatibility. Memory \& Cognition, 7, 375-381.

Klapp, S. T., Hill, M. D., Tyler, J. G., Martin, Z. E., Jagacinski, R. J., \& Jones, M. R. (1985). On manching to two different drummers: Perceptual aspects of the difficulties. Journal of Experimental Psychology: Human Perception and Performance, 11, 814-827.

Klapp, S. T., Nelson, J. M., \& Jagacinski, R. J. (1998). Can people tap concurrent bimanual thythms independently? Journal of Motor Behavior, 30, 301-322.

Krampe, R. T., \& Ericsson, K. A. (1996). Maintaining excellence: Cognitive-motor performance in pianists differing in age and skill level. Journal of Experimental Psychology: General, 125, 331-359.

MacKay, D. G. (1987). The organization of perception and action: A theory for language and other cognitive skills. New York: Springer-Verlag.

Peper, C., Beek, P. J., \& van Wieringen, P. C. W. (1995). Multifrequency coordination in bimanual tapping: Asymmetrical coupling and signs of supercriticality. Journal of Experimental Psychology: Human Perception and Performance, 21, 11171138.

Peters, M. (1981). Attentional asymmetries during concurrent bimanual performance. Quarterly Journal of Experimental Psychology: Human Experimental Psychology, 33A, 95-103.

Peters, M., \& Schwartz, S. (1989). Coordination of the two hands and effects of attentional manipulation in the production of a bimanual 2:3 polyrhythm. Australian Journal of Psychology, 41, 215-224.

Povel, D. (1981). Internal representations of simple temporal patterns. Journal of Experimental Psychology: Human Perception and Performance, 7, 3-18.

Pressing, J. (1995). Testing dynamical and cognitive models of rhythmic pattern production. In D. J. Glencross \& J. P. Piek 
(Eds.), Motor control and sensory motor integration: Issues and directions (pp. 141-170). Amsterdam: Elsevier Science.

Pressing, J., Summers, J., \& Magill, J. (1996). Cognitive multiplicity in polynythmic pattern performance. Joumal of Experimental Psychology: Human Perception and Performance, 22, 1127-1148.

Repp, B. (1999). Control of expressive and metronomic timing in pianists. Journal of Motor Behavior, 31, 145-164.

Salthouse, T. A. (1991). Expertise as the circumvention of human processing limitations. In K. A. Ericsson \& J. Smith (Eds.), Toward a general theory of expertise: Prospects and limits (pp. 286-300). New York: Cambridge University Press.

Shaffer, L. H. (1981). Performances of Chopin, Bach, and Bartok: Studies in motor programming. Cognitive Psychology, 13, 326-376.

Summers, J. J., Ford, S. K., \& Todd, J. A. (1993). Practice effects on the coordination of the two hands in a bimanual tapping task. Human Movement Science, 11, 101-112.

Summers, J. J., \& Kennedy, T. M. (1992). Strategies in the production of a 5:3 polyrhythm. Human Movement Science, 11, 101-112.

Summers, J., \& Pressing, J. (1994). Coordinating the two hands in polyrhythmic tapping. In S. P. Swinnen, H. Heuer, J. Massion, \& P. Casaer (Eds.), Interlimb coordination: Neural, dynamical, and cognitive constraints (pp. 571-593). San Diego, CA: Academic Press.

Summers, J. J., Rosenbaum, D. A., Burns, B. D., \& Ford, S. K. (1993). Production of polyrhythms. Journal of Experimental Psychology: Human Perception and Performance, 19, 416-428.

Turvey, M. T., Schmidt, R. C., \& Rosenblum, L. D. (1989). "Clock" and "motor" components in absolute coordination of rhythmic movements. Neuroscience, 33, 1-10.

Vorberg, D., \& Hambuch, R. (1978). On the temporal control of rhythmic performance. In J. Requin (Ed.), Attention and performance VII (pp. 535-555). Hillsdale, NJ: Erlbaum.

Vorberg, D., \& Hambuch, R. (1984). Timing of two-handed rhythmic performance. Annals of the New York Academy of Sciences, 423, 390-406.

Vorberg, D., \& Wing, A. M. (1996). Modelling variability and dependence in timing. In H. Heuer \& S. W. Keele (Eds.), Handbook of perception and action: Vol. 3. Motor skills (pp. 181-261). London: Academic Press.

Wing, A. M. (1977). Perturbations of auditory feedback delay and the timing of movement. Journal of Experimental Psychology: Human Perception and Performance, 3, 175-186.

Wing, A. M. (1980). The long and the short of timing in response sequences. In G. E. Stelmach \& J. Requin (Eds.), Tutorials in motor behavior (pp. 469-486). Amsterdam: North-Holland.

Wing, A. M. (1982). Timing and coordination of repetitive bimanual movements. Quarterly Journal of Experimental Psychology: Human Experimental Psychology, 34A, 339348.

Wing, A. M., Church, R. M., \& Gentner, D. R. (1989). Variability in the timing of responses during repetitive tapping with altemate hands. Psychological Research, 51, 28-37.

Wing, A. M., \& Kristofferson, A. B. (1973a). Response delays and the timing of discrete motor responses. Penception \& Psychophysics, $14,5-12$.

Wing, A. M., \& Kristofferson, A. B. (1973b). The timing of interresponse intervals. Perception \& Psychophysics, 13, 455-460.

Yamanishi, J., Kawato, M., \& Suzuki, R. (1980). Two coupled oscillators as a model for the coordinated finger tapping in both hands. Biological Cybernetics, 37, 219-225.

\section{Appendix}

\section{Covariance Modeling: Derivations and Examples}

\section{Definitions for Variance and Covariance}

For arbitrary random variables $X$ and $Y$ with expected values (means) $E(X)$ and $E(Y)$, variance and covariance are defined as follows:

Definition 1: $\sigma_{X}^{2}=\operatorname{var}(X)=E\left(X^{2}\right)-E^{2}(X)$,

Definition 2: $\operatorname{cov}(X, Y)=E(X Y)-E(X) E(Y)$.

Note that, by definition, $\operatorname{var}(X)=\operatorname{cov}(X, X)$. Let $X=a U+b V$ and $Y=c W+d Z$, where $U, V, W$, and $Z$ are random variables and $a, b, c$, and $d$ are arbitrary constants. From the above definitions, the distributivity of covariances is as follows:

$$
\begin{aligned}
& \operatorname{cov}(X, Y) \\
& =\operatorname{cov}(a U+b V, c W+d Z) \\
& =a c \operatorname{cov}(U, W)+a d \operatorname{cov}(U, Z)+b c \operatorname{cov}(V, W) \\
& \quad+b d \operatorname{cov}(V, Z) .
\end{aligned}
$$

\section{Covariances of Sums and Differences}

Using Equation Al, we find

$$
\begin{aligned}
\operatorname{var}(U+V) & =\operatorname{cov}(U+V, U+V) \\
& =\operatorname{var}(U)+2 \operatorname{cov}(U, V)+\operatorname{var}(V)
\end{aligned}
$$

Similarly,

$$
\begin{aligned}
\operatorname{var}(U-V) & =\operatorname{cov}(U-V, U-V) \\
& =\operatorname{var}(U)-2 \operatorname{cov}(U, V)+\operatorname{var}(V) .
\end{aligned}
$$

\section{Rate Fluctuation}

Consider two intervals $U=A X$ and $V=B X$, each of which is specified as the product of an interval specified on some internal scale multiplied by the target period duration $X . A, B$, and $X$ are random variables, and $X$ is assumed to be independent of $A, B$, and of the product $A B$. 
$\operatorname{cov}(U, V)$

$$
\begin{aligned}
= & \operatorname{cov}(A X, B X) \\
= & E[(A X)(B X)]-E(A X) E(B X) \quad \text { (by Definition 2) } \\
= & E(A B) E\left(X^{2}\right)-E(A) E(B) E^{2}(X) \quad \text { (by independence) } \\
= & E(A B) E\left(X^{2}\right)-E(A) E(B) E^{2}(X) \\
& +E(A) E(B) E^{2}(X)-E(A) E(B) E^{2}(X) . \\
= & {[E(A B)-E(A) E(B)] E\left(X^{2}\right)+E(A) } \\
& +E(B)\left[E\left(X^{2}\right)-E^{2}(X)\right] \\
= & \operatorname{cov}(A, B) E^{2}(X) \quad \text { (by Definitions } 1 \text { and 2) } \\
& +E(A) E(B) \text { var }(X) . \quad
\end{aligned}
$$

Equation A4 shows that the covariances between intervals depend on the amount of rate fluctuation, that is, the variance of $X$ and the product of the mean proportions $E(A)$ and $E(B)$ specified in the rhythm program. If $A$ and $B$ are uncorrelated, the first term in Equation A4 vanishes for all covariance between different intervals. This does not hold for the variances of target intervals because

$$
\operatorname{cov}(A X, A X)=\operatorname{var}(A) E^{2}(X)+E^{2}(A) \operatorname{var}(X) .
$$

In our modeling approaches throughout the article, we assume constant proportions (i.e., $A=a$, and $B=b$ ). Under these assumptions, the first term in Equation A5 likewise vanishes. Theoretically, this assumption amounts to perfect propagation of proportions, which is plausible for models with only one hierarchy level like ours. At rapid tempos, variations in the proportions realized within a trial were small in the first place.

\section{Covariances Predicted by the Parallel Model}

By applying the distributivity of covariances, we derive the predictions for the parallel model. For simplicity, we omit the contributions of the motor delay here. As an example, consider the covariance between intervals $\mathrm{I}_{2}$ and its successors. The expected (mean) intervals in the first line of each prediction are listed in
Table 3. We use small letters $a, b, c, d$, and $e$ to denote the proportions of $X$ controlled by timekeepers A, B, C, D, E, and F, respectively.

$$
\begin{aligned}
c_{23}= & \operatorname{cov}\left(e X-a X+I_{0}, a X+b X-e X-I_{0}\right) \\
= & \operatorname{cov}(e X-a X, a X+b X-e X)-\operatorname{var}\left(\mathrm{L}_{0}\right) \\
= & \operatorname{cov}(e X, a X)+\operatorname{cov}(e X, b X) \\
& -\operatorname{cov}(e X, e X)-\operatorname{cov}(a X, a X)-\operatorname{cov}(a X, b X) \\
& +\operatorname{cov}(a X, e X)-\operatorname{var}\left(\mathrm{I}_{0}\right) \\
= & {[e(a+b-e)-a(a+b-e)] } \\
& \cdot \operatorname{var}(X)-\operatorname{var}\left(\mathrm{I}_{0}\right) . \quad \text { (by A4 and A5) }
\end{aligned}
$$

In the more general case, expressions including the variances of proportions for those timekeepers contributing to both intervals appear (see Equation AS), that is, $\operatorname{var}(A)$ and $\operatorname{var}(E)$ in our example. As stated above, these expressions vanish if we assume constant proportions within a given trial:

$$
\begin{aligned}
c_{24}= & \operatorname{cov}\left(e X-a X+I_{0}, e X+f X-a X-b X+I_{0}\right) \\
= & {[e(e+f-a-b)-a(e+f-a-b)] } \\
& \cdot \operatorname{var}(X)+\operatorname{var}\left(\mathbf{I}_{0}\right) .
\end{aligned}
$$

Substituting the sums in brackets by (ideal) values for the proportions (i.e., $a=b=c=d=1 / 4 ; e=f=1 / 3$ ), we obtain the identical predictions for the positive covariation introduced by rate fluctuation (see Table 4) as in the integrated model (see Table 1). The critical difference between the models is the prediction of positive and negative covariances related to $\sigma_{0}^{2}$ in the parallel model. Note also that $\mathrm{c}_{26}$ only depends on rate fluctuation and not on $\sigma_{0}^{2}$.

$$
c_{26}=\operatorname{cov}\left(e X-a X+\mathrm{I}_{0}, d X\right)=[(e-a) d] \operatorname{var}(X) .
$$

Received January 6, 1997

Revision received August 24, 1998 Accepted March 4, 1999 